\title{
Les eaux d'irrigation du Haouz de Marrakech : un siècle de confrontations des modèles de gestion publics, privés et communautaires
}

\section{Thierry Ruf and Mina Kleiche-Dray}

\section{(2) OpenEdition}

Journals

Electronic version

URL: https://journals.openedition.org/echogeo/15258

DOI: $10.4000 /$ echogeo. 15258

ISSN: 1963-1197

Publisher

Pôle de recherche pour l'organisation et la diffusion de l'information géographique (CNRS UMR 8586)

Electronic reference

Thierry Ruf and Mina Kleiche-Dray, "Les eaux d'irrigation du Haouz de Marrakech : un siècle de confrontations des modèles de gestion publics, privés et communautaires", EchoGéo [Online], 43। 2018, Online since 22 March 2018, connection on 23 August 2021. URL: http:// journals.openedition.org/echogeo/15258; DOl: https://doi.org/10.4000/echogeo.15258

This text was automatically generated on 23 August 2021.

EchoGéo est mis à disposition selon les termes de la licence Creative Commons Attribution - Pas d'Utilisation Commerciale - Pas de Modification 4.0 International (CC BY-NC-ND) 


\section{Les eaux d'irrigation du Haouz de Marrakech : un siècle de confrontations des modèles de gestion publics, privés et communautaires}

Thierry Ruf and Mina Kleiche-Dray

\section{AUTHOR'S NOTE}

À la mémoire de Feu Pr Mohammed El Faiz, inspirateur de nombreuses recherches sur l'histoire hydraulique du Maroc.

1 Le débat sur le modèle de gestion bureaucratique de l'irrigation a débuté dans les années 1960 au Maroc dans le cadre des politiques de développement qui suivent l'Indépendance. À cette époque, à Marrakech, le sociologue Paul Pascon est au cœur des questions hydrauliques, en tant que directeur du tout nouvel office régional de mise en valeur agricole du Haouz ${ }^{1}$ (ORMVAH). Au terme de son mandat, il écrit en 1968 une note de travail dans laquelle il s'interroge sur la bonne voie à suivre pour les irrigants. Fautil continuer le processus de grande hydraulique dont la bureaucratie constitue une charge trop lourde, autant pour l'État que pour les paysans? Faut-il au contraire organiser une décentralisation de la gestion de l'eau à des associations locales d'usagers avec un minimum de contrôle gouvernemental (Pascon, 1968) ? Ces questions renvoient directement aux théories du développement de l'hydraulique et à celle de l'accès aux ressources naturelles: en sciences politiques, Karl Wittfogel considère que le despotisme est nécessaire à l'émergence d'une économie hydraulique supérieure (Wittfogel, 1957). Dans les pays en développement, de nombreux échecs des bureaucraties hydrauliques mises en place après les indépendances sont constatés. Dans les années 1970, l'école anglo-saxonne des commons, incarnée notamment par 
Elinor Ostrom, rejette autant le principe de supériorité de la gestion étatique de Wittfogel que celui de la tragédie des communs qu'avait soutenu Hardin en 1968, en appelant à la privatisation des accès aux ressources naturelles (Ruf, 2011). Ostrom défend un ensemble de principes de gestion communautaire de l'irrigation considérés comme indispensables pour réformer le secteur administratif public de l'eau (Ostrom, 1992).

2 Au Maroc, dans ces mêmes années 1970, Paul Pascon, retourné au monde de la recherche et de l'université, soutient une thèse d'État de sociologie, le Haouz de Marrakech, histoire sociale et structures agraires (Pascon, 1977). À la fermeture du Centre de Sociologie de l'Université Mohamed V, il développe ses travaux au sein du département des sciences humaines de l'Institut Agronomique et Vétérinaire Hassan II. Il y forme de nombreux chercheurs à la sociologie rurale dans une visée de rechercheaction en rupture avec la vision techniciste du développement agricole et rural dans laquelle s'était engagé le gouvernement marocain à partir de la fin des années $1960^{2}$. On retiendra ici des travaux de Paul Pascon la construction du concept de société composite " pour décrire une société marocaine dominée par un mélange de modèles de "patriarcat », " tribalisme ", "féodalisme » et "capitalisme " $^{3}$ dont l'agencement est complexe, évolutif avec des relations issues de compromis historiques et de compétitions économiques et politiques. D'un côté, ces dernières favoriseraient le renforcement du capitalisme, du fait des emprunts technologiques menant peu à peu aux reculs des coutumes, el 'urff. D'un autre côté, le caractère hydraulique de l'économie du Haouz offre un monde plus complexe marqué par des aléas climatiques et des risques anthropiques et politiques qui ne donnent pas de garanties suffisantes pour les investisseurs. Le développement du Haouz passe-t-il par le renforcement du modèle makhzénien ${ }^{5}$ d'étatisation de la distribution de l'eau ou bien par une certaine transition capitaliste toujours enchâssée dans l'enchevêtrement des relations sociales composites dans la région du Haouz? La «question hydraulique » fait alors l'objet de recherches nouvelles sur la coexistence de deux grandes formes d'organisation des réseaux, la Grande Hydraulique (GH) d'une part, associée à la création des grands barrages et sous contrôle des offices régionaux de mise en valeur agricole, le modèle makhzénien (Popp 1984) et, la PMH, la Petite et Moyenne Hydraulique, regroupant tout le reste sous la dépendance administrative des Directions provinciales de l'agriculture (Bouderbala et al., 1984).

3 Quarante ans ont passé et le contexte à la fois national et international de conflits sur le foncier, sur l'exploitation intensive des ressources naturelles, sur la non-prise en compte des savoirs localisés, continue d'interroger les possibilités de mise en place d'un système de partage des eaux durable et équitable. Il nous semble alors que ce recul permet de revisiter la question sociale et hydraulique du Haouz pour aborder, dans l'ensemble régional comme dans différents territoires hydrauliques, les évolutions et les recompositions autour des accès, des partages et des usages des eaux. L'objectif de notre article est de saisir les recompositions territoriales spatiales et historiques des réseaux anciens et modernes face au patchwork hydraulique et social hérité en analysant à la le fonctionnement actuel de différents systèmes hydro-agricoles avec leurs diverses communautés d'irrigants tout en les ancrant dans l'histoire de ces aménagements et de leurs usages entremêlés. Pour cela nous allons analyser les différentes trajectoires prises par l'office du Haouz décrites par Paul Pascon grâce au corpus d'archives de l'ORMVAH dont nous avons dressé l'inventaire depuis 2001. En 
effet, L'ORMVAH existe toujours en 2018, en tant qu'opérateur public de l'eau agricole de la région, un office qui perpétue le modèle makhzénien de gestion de l'eau et avec qui notre groupe de recherche a travaillé durant la décennie 2000 (Ruf, 2009) puis plus récemment dans les années 2016-2018. Ce corpus a été complété par les documents consultés au sein du service de gestion de l'irrigation et du drainage à Marrakech, des archives de l'Armée de Vincennes et de Saint Denis et des Archives diplomatiques de Nantes, très riches sur le Maroc, même si elles sont moins fournies sur la région du Haouz. La méthodologie que nous avons adoptée est un suivi des différentes trajectoires singulières de ces aménagements sur un siècle d'histoire de confrontation entre les tenants d'une gestion centralisée et imposée, ceux d'une gestion néolibérale et ceux d'une gestion communautaire. Nous verrons ainsi au cas par cas quels ont été les compromis construits entre ces différents modèles, et comment ceux-ci perpétuent des reconfigurations toujours composites des sociétés humaines en tension permanente lorsque l'hydraulique conditionne l'existence même de ces sociétés locales.

La ville de Marrakech, principale cité de la région du Haouz, et ancienne capitale du Maroc, et ses environs disposent d'aménagements hydrauliques conséquents depuis le XII ${ }^{e}$ siècle, à la fois dans le cadre urbain de la cité - jardin que dans celui de la palmeraie qui l'entoure sur plusieurs dizaines de milliers d'hectares ${ }^{6}$. Fort de cette hydraulique mêlant à la fois des réseaux de surface et des captages d'eau souterraine via de très nombreuses galeries drainantes, Marrakech devient à la fin du XIX ${ }^{\mathrm{e}}$ siècle une région attractive pour des projets européens de colonisation (illustration 1). Le Haouz de Marrakech était considéré par les courtiers européens comme une excellente place pour y développer la production de coton (Pascon, 1983, t. 2, p. 517). Avec l'intention d'entrer en concurrence avec la production cotonnière égyptienne, des sociétés privées et des particuliers établissent, dans les premières années du $\mathrm{XX}^{\mathrm{e}}$ siècle, différentes fermes en s'associant localement avec l'élite des caïds. Ils expérimentent des technologies et des spéculations nouvelles. Il est évident que le climat est alors favorable aux cultures industrielles malgré la restriction de la disponibilité en eau et sans doute également, malgré les incertitudes sur les régimes fonciers et sur les formes juridiques d'accès à l'eau. La situation était en effet confuse du fait d'une longue tradition d'expulsion et de réinstallation de différents groupes de populations regroupés autour des systèmes tribaux ${ }^{7}$ en fonction de leur soumission ou de leur opposition aux élites politiques au pouvoir au Maroc (Pascon, 1983, t. 1, p. 152). 


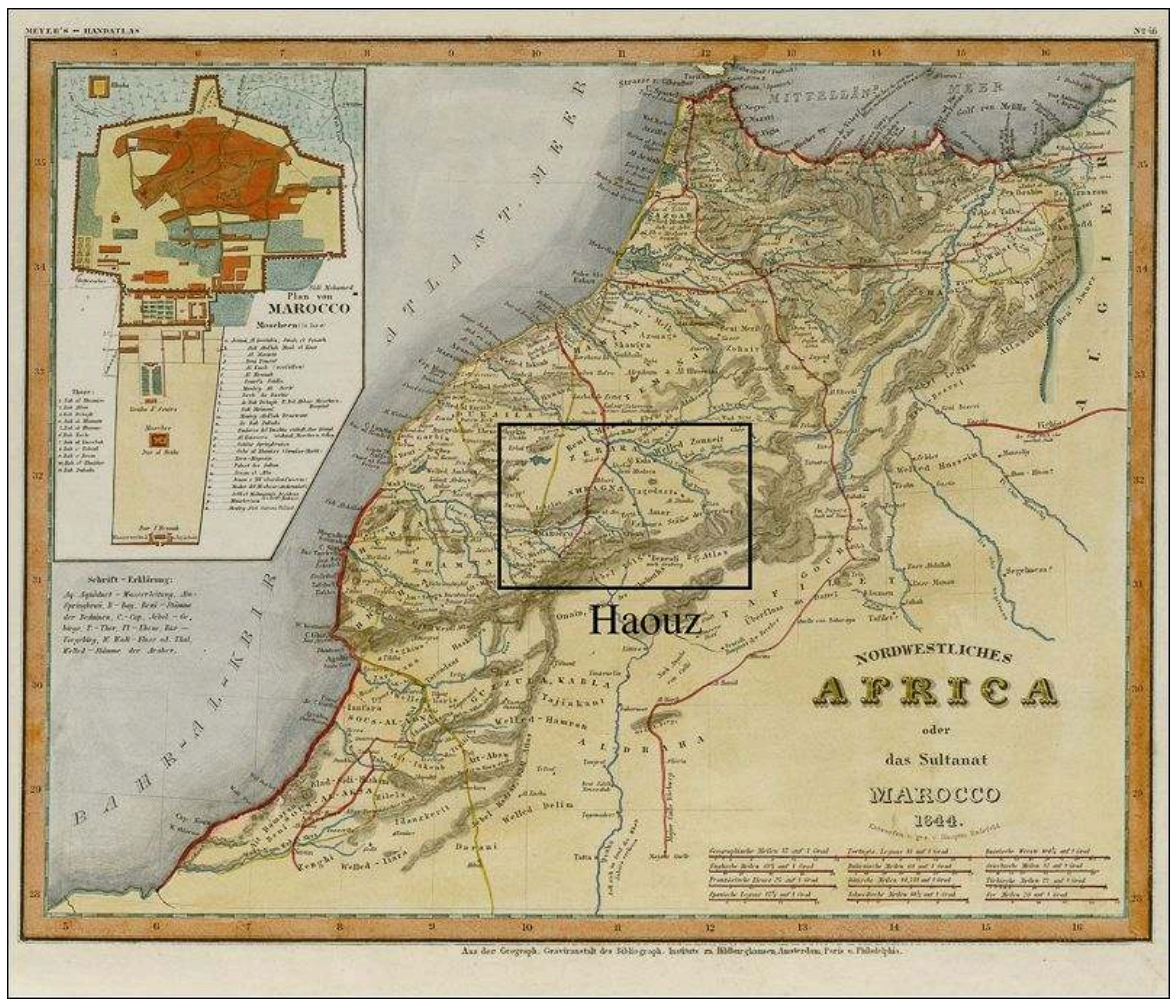

Marrakech est alors un lieu bien connu du développement hydraulique issu des initiatives araboandalouses du Moyen Âge. Deux hauts bassins hydrographiques principaux contribuent à alimenter la région du Haouz : le Tensift et l'Oum Ar Rabia.

Sources : Meyer Joseph, 1860. Nordwestliches Africa oder das Sultanat Marocco, 1844. Aus der Geograph. Graviranstalt des Bibliograph. Instituts zu Hildburghausen, Amsterdam, Paris u. Philadelphia

\section{Le modèle étatique colonial d'ingénierie de l'eau au temps du protectorat (1912-1955)}

La période coloniale commence formellement au Maroc en 1912. Une des premières décisions consiste à retirer à la fondation des $\mathrm{Haboûs}^{8}$ toute compétence en matière d'eau et de promulguer la domanialité de l'eau dont l'État prend la charge des attributions et de la gestion (Dahir du 7 Chaabane $1332-1^{\mathrm{er}}$ juillet 1914$)^{9}$. De nouveaux acteurs du pays, les administrateurs - ingénieurs de l'eau débutent très tôt la mission d'aménagement hydraulique coloniale. Les documents d'archives, consultés au siège de l'Office régional de mise en valeur du Haouz, montrent un intérêt très précoce des fonctionnaires français pour décrire l'ensemble des captages, canaux (seguias) et galeries drainantes (khettaras) dans le tissu urbain et son environnement (Kleiche-Dray, 2007). Durant toute la période de 1912 à 1955 , ils vont diviser le monde de l'eau en deux ensembles qui préfigurent en partie la dichotomie future entre la Petite et Moyenne Hydraulique et la Grande Hydraulique.

D'un côté, le monde ancien des indigènes ${ }^{10}$ est abordé sous l'angle de la tradition. Ici, ce qui est en jeu est la reconnaissance des anciens prélèvements d'eau mais en en les fixant à partir de mesures systématiques journalières des débits dans les oueds et les canaux. En pratique, après quelques années de mesures, les ingénieurs proposent une 
matrice de droits d'eau en fonction de l'abondance ou de la rareté de l'eau dans l'oued. Cela signifie qu'aux moments des étiages, le droit indigène reconnu est très restreint. En cas d'apport d'eau supplémentaire, le débit supérieur à celui reconnu pour les indigènes servira d'autres usagers.

De l'autre côté, le monde moderne des colons européens bénéficient de nouveaux droits d'eau en débits continus prélevés sur les rivières et les sources, puis sur les réserves créées grâce aux premiers barrages ou sur les stocks d'eau souterraine (alors en accès illimité et concurrentiel vis-à-vis des khettaras vives gérées le plus souvent par les indigènes eux-mêmes).

En fait, les archives de l'ORMVAH montrent que, juste après l'instauration du protectorat, les services hydrauliques français dressent un inventaire des centaines de khettaras avec un très grand niveau de précision en topographie, description des composantes des ouvrages et mesure des débits caractéristiques.

Illustration 2 - Un exemple de la description précoce des systèmes hydrauliques à Marrakech. La galerie drainante de Ain Sidi Moussa, 1917

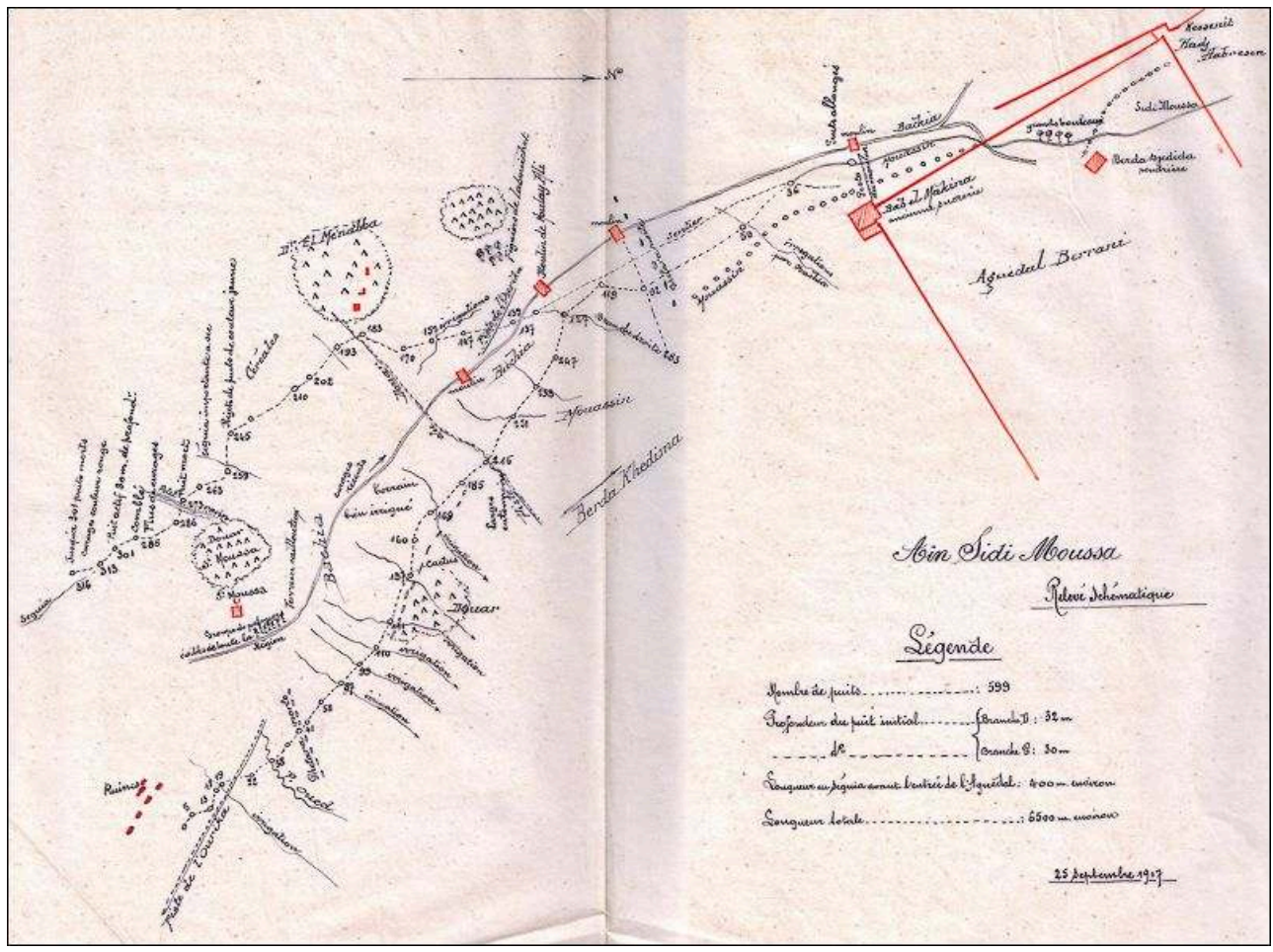

Source : Archives ORMVAH, Marrakech.

9 À titre d'exemple, parmi les archives de cet inventaire, figure le dossier de l'Ain Sidi Moussa et son plan détaillé dressé le 25 septembre 1917 (illustration 2). Bien qu'Ain signifie source en arabe, il s'agit d'une galerie drainante de 6500 mètres de long comprenant 599 puits de visite. La khettara commence à capter l'eau souterraine au niveau des puits-mère de deux branches, à plus de 30 mètres sous le niveau du sol. Les deux branches se réunissent puis l'eau converge vers le vaste jardin Berrani où elle circule en surface quelques 400 mètres avant de passer sous l'enceinte du jardin. L'origine de l'eau est certainement composite. Une des branches est décrite avec la mention d'une trentaine de puits sans eau dans la partie la plus en amont, puis l'apparition d'une zone drainante de la nappe. La galerie suit d'assez près un canal qui 
traverse aussi le jardin Berrani. Cette seguia devait contribuer par ses pertes à fournir de l'eau à la khettara Ain Sidi Moussa. Par ailleurs, le schéma donne aussi des informations complémentaires sur d'autres vecteurs hydriques, les oueds, les khettaras vives ou mortes qui avoisinent la galerie décrite, et les lieux où on pratique l'irrigation à proximité et qui sont aussi des pourvoyeurs d'eau perdue dans l'arrosage des champs.

L'utilité de ce premier inventaire n'est pas explicite. Il révèle plutôt une fascination des ingénieurs coloniaux qui semblent découvrir comment la Medina de Marrakech et la palmeraie qui l'entoure dépendent totalement de cet ensemble ingénieux. L'approche reste cependant très monographique sans prise en compte de l'arrangement spatial de l'ensemble des galeries. Quelques années après, les services hydrauliques systématisent la localisation de l'ensemble des khettaras et définissent une sectorisation administrative du suivi hydraulique, en vue de quantifier les débits journaliers et d'inclure les khettaras dans la politique de reconnaissance des droits d'eau, comme cela se pratique sur les eaux de surface. La carte des attributions des blocs de surveillance du réseau hydraulique, non datée précisément mais probablement dressée dans les années 1930 (illustration 3), montre que l'administration coloniale organise avec minutie le recueil des informations sur les ouvrages, les bénéficiaires et les débits qui transitent aussi bien en surface que par le réseau de plusieurs centaines de khettaras. Les 624 galeries drainantes surveillées représentaient 800 kilomètres de "vecteurs hydrauliques". S'y ajoutaient 39 seguias, 12 pompages électriques et plus de 200 pompages ordinaires, vraisemblablement des norias actionnées par des animaux de traction.

Illustration 3 - Plan illustrant la sectorisation et le suivi des centaines de khettaras de la ville et des environs de Marrakech vers 1940

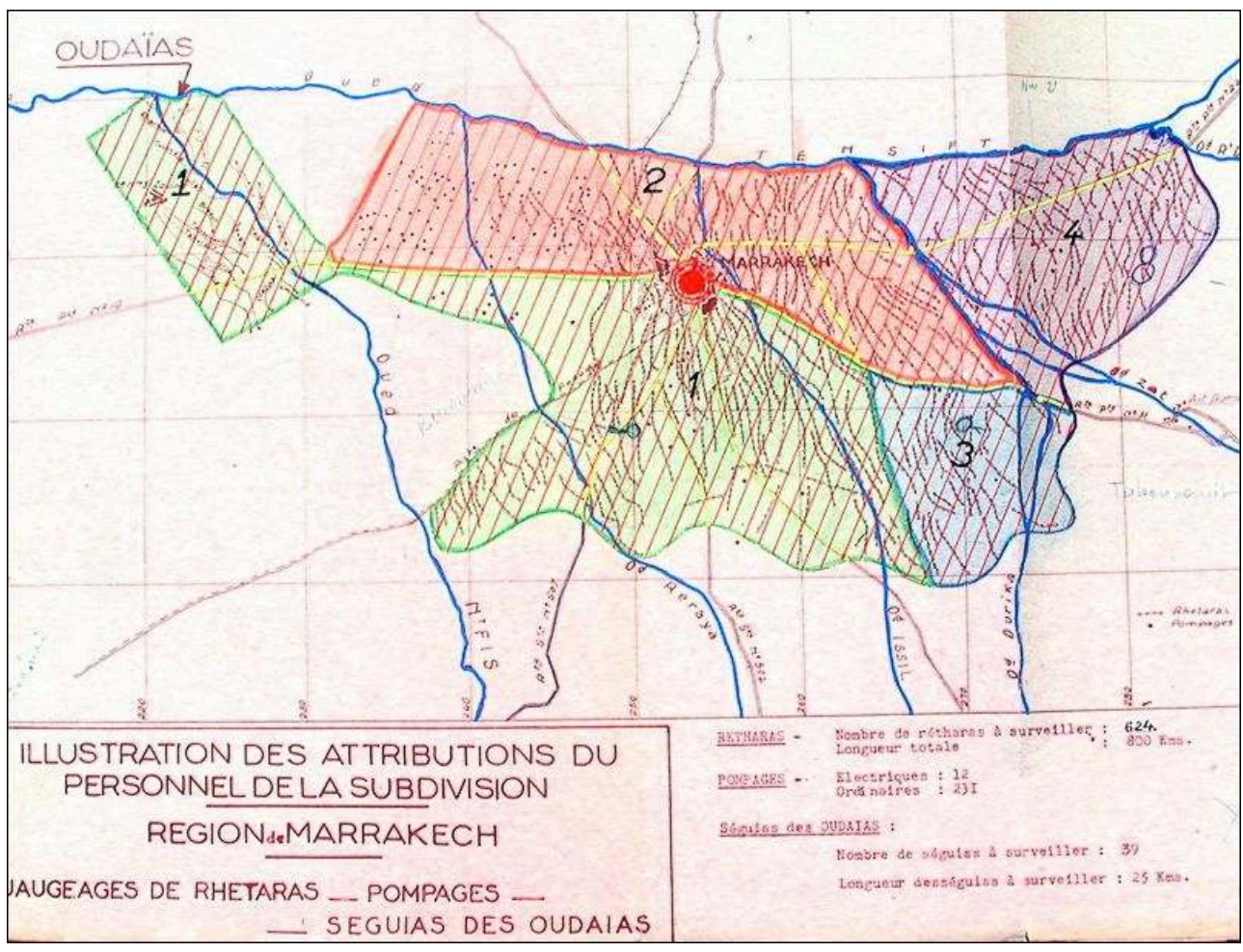

Source : Archives ORMVAH, Marrakech. 
11 Les galeries drainantes n'étaient pas toutes privées ou individuelles. Quelques-unes parmi les plus importantes relevaient de la ville de Marrakech ou de l'État lui-même qui disposait des grands jardins en périphérie proche de la Médina. En effet, l'armée marocaine a joué un rôle éminent dans l'aménagement hydraulique du Haouz dès le XII siècle et jusqu'à la colonisation (Bouderbala, 1987, 1999). Une très grande proportion des terres irrigables du Haouz de Marrakech relève d'ailleurs encore aujourd'hui du statut Guich $^{11}$. D'autres khettaras étaient aux mains de petites communautés paysannes co-usagers de l'eau recueillie.

Les recherches dans les archives de l'ORMVAH indiquent que plusieurs colons européens avaient aussi sous leur contrôle des khettaras anciennes associées à leur ferme, mais ils en avaient parfois aussi créées eux-mêmes comme le révèlent diverses demandes d'autorisation administrative. Les services hydrauliques intervenaient lorsqu'éclataient des conflits entre usagers au moment de réduction des débits, et parfois, proposaient des aides pour assurer leur maintenance. Au cours d'une des visites aux archives en 2004, nous avons repéré sur une pile de documents, en instance de rangement, une note écrite vers 1950 par un ingénieur français qui alertait ses supérieurs hiérarchiques sur la crise hydrique proche que trente années de jaugeages des débits permettaient de comprendre. Le tarissement progressif des galeries drainantes était perceptible et selon lui, imputable directement à la surexploitation de la nappe phréatique dans les grandes fermes européennes de la frange nord du Haouz central arrosé plutôt par les khettaras, et même à l'intérieur de la zone irriguée. Malheureusement, le lendemain, cette pile de documents fut déplacée et la note égarée et perdue au milieu de très nombreuses liasses de papiers.

L'administration coloniale n'a évidemment pas seulement inventorié et surveillé les galeries drainant les eaux souterraines. Elle a cherché à construire une connaissance exhaustive de tous les canaux de surface et établi un plan pour déterminer l'ensemble des débits des rivières, des débits prélevés par les canaux à leur prise et parfois aussi dans leurs ramifications. L'argument avancé pour développer cette ingénierie de la mesure des flux d'eau était l'existence de conflits permanents entre les groupes indigènes eux-mêmes et, sans aucun doute aussi, entre communautés indigènes gérant des seguias et exploitants européens cherchant à accéder soit aux canaux existants soit prêts à en construite de nouveaux pour leur usage exclusif.

Depuis le début du protectorat, le cadre juridique et politique de l'eau avait changé : avec la déclaration donnant à l'eau le caractère de bien public, la nouvelle administration avait acquis une légitimité pour traiter des questions de régulation de l'eau et de refonte des droits d'accès. Ainsi, de très nombreuses missions hydrauliques ont travaillé avec une méthodologie commune pour rassembler une quantité d'informations impressionnante pour chaque dispositif hydraulique du Maroc. En 1936, la mission hydraulique de Marrakech publie une note guidant la mise en place des jaugeages en des points précis des cours d'eau, avec les recommandations pratiques pour l'établissement des mesures (illustration 4). 
Illustration 4 - Jaugeages

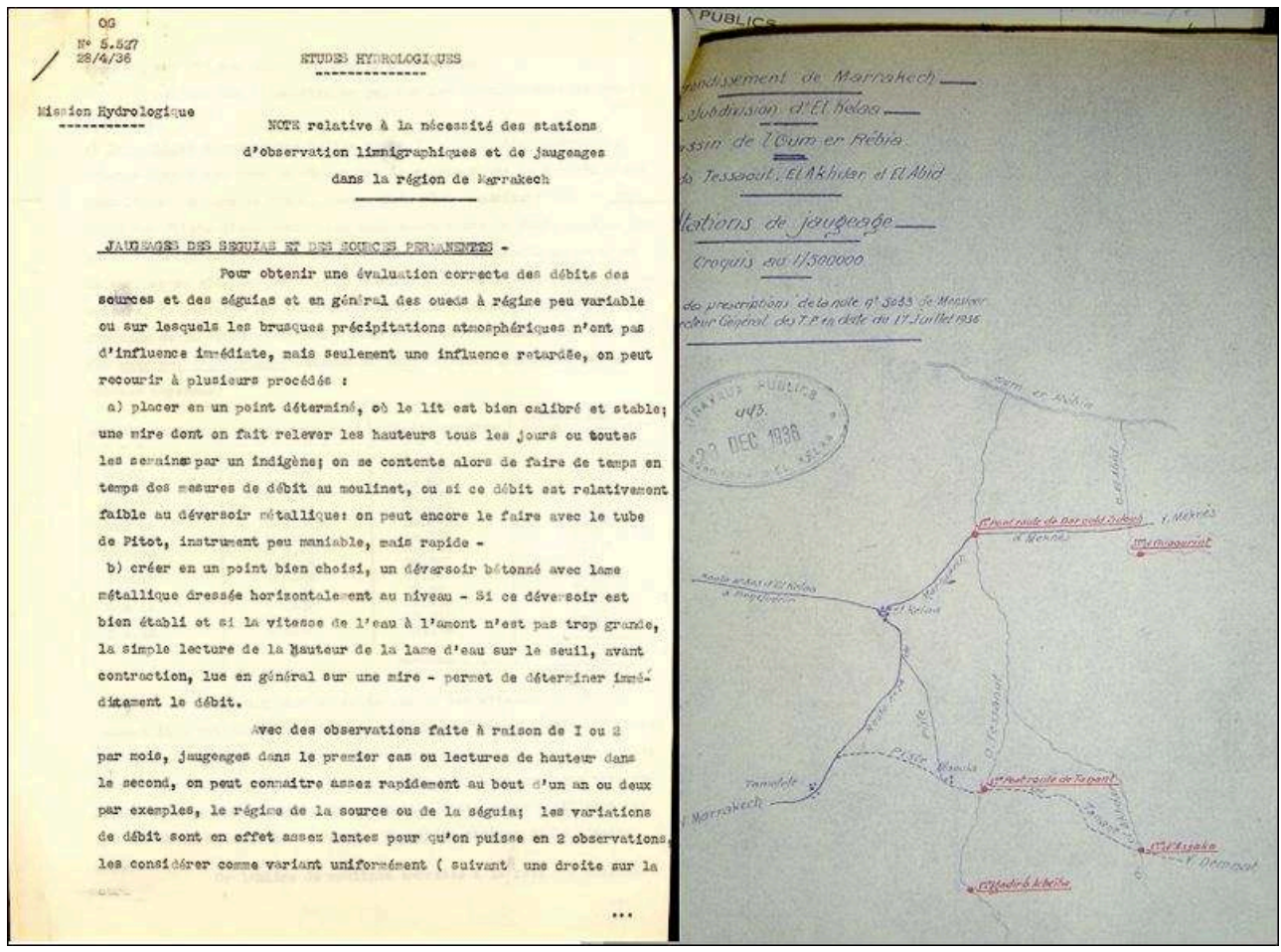

Les ressources en eau sont étudiées à travers un système de mesures dont l'objectif est de définir les droits d'eaux des « indigènes » et les marges possibles pour l'installation de colons " européens ».

Source : Archives ORMVAH, Marrakech, 1936

Partout, des dispositions sont prises pour mesurer les débits en des lieux propices, souvent liés aux ponts des routes ou pistes principales, comme on peut le voir sur la carte des stations de jaugeage de l'oued Tessaout au Nord Est de Marrakech. En parallèle, des campagnes de mesures des débits dérivés dans les canaux traditionnels sont lancées. Le but apparent premier de ces opérations est de quantifier les accès aux eaux des indigènes dans le cadre des régimes «naturels » des rivières, en retenant ce qui se passe lors des étiages, et de proposer alors une actualisation des droits anciens susceptible de pacifier les conflits de l'eau. Le but principal consiste en fait à définir quels sont les droits d'eau qui pourraient être attribués à des Européens si on établit des barrages - réservoirs sur ces rivières. Les indigènes resteraient avec leur seuls droits d'étiages et les colons seraient bénéficiaires des lames d'eau supplémentaires lors des lâchers de barrage. Malgré tout cet appareillage, plusieurs facteurs contribuent à l'imprécision générale des données recueillies et des traitements effectués. D'une part, les méthodologies retenues varient beaucoup d'un point de mesure à un autre, et reposent en grande partie sur des interventions humaines. La conversion des hauteurs d'eau en débits a toujours été un point délicat en hydrologie pratique. D'autre part, la variabilité climatique est particulièrement forte au Maroc et singulièrement dans le Haouz de Marrakech, et il faudrait disposer de chroniques de mesures sur au moins trois décennies pour commencer à cerner véritablement les différents scenari hydriques. Or, l'administration coloniale va utiliser les données mesurées presque immédiatement, avec seulement quelques années de données disponibles mais pas toutes cohérentes et vérifiées. Enfin, et c'est sans doute un point crucial, l'artificialisation des rivières existe depuis les hauts bassins montagnards jusqu'aux plaines d'aval. Des centaines d'ouvrages prélèvent de l'eau et modifient les écoulements 
naturels, mais pas de la même façon chaque année et même chaque mois. Il faut ajouter à ce panorama des approximations tous les phénomènes méconnus des liens entre les eaux de surface et les eaux souterraines, l'impact des infiltrations et des résurgences.

Pour contrôler les différents flux et proposer une nouvelle régulation fondée sur des données scientifiques, les ingénieurs coloniaux recourent à différentes représentations géographiques. Ainsi, ils firent dresser de nombreuses cartes des systèmes hydrauliques locaux et établir des documents synthétiques comme la carte générale des oueds et canaux de surface du Haouz de Marrakech publiée vers 1940 (illustration 5).

Ce document montre la très forte densité de canaux sur l'ensemble des sept rivières principales descendant du Haut Atlas. La carte se limite aux seuls ouvrages irrigant la plaine. Or, il faut noter qu'en montagne, les hauts bassins versants de chaque rivière ont aussi des dizaines, voire des centaines de canaux de dérivation, certes d'ampleur moindre pris séparément, mais non négligeable pris dans leur totalité. Une partie de ces ouvrages a été remaniée, une autre a disparu avec la reconfiguration hydraulique de la fin du XXe siècle, mais dans l'ensemble, les seguias existent toujours aujourd'hui et continuent de dériver des eaux de crues et des eaux pérennes, notamment à l'endroit des résurgences. La carte montre aussi que l'allocation des eaux ne se raisonne pas uniquement dans chaque bassin versant sans tenir compte des autres. En réalité, les accès à l'eau ne pouvaient pas être compris par l'identification des seuls lieux de prélèvements des eaux mais en regardant les espaces qui en recevaient les bénéfices, souvent situés en dehors du bassin versant stricto sensu, dans les interfluves parfois très éloignées du bassin d'origine de l'eau dérivée : nous avons dénommé bassins déversants ces espaces de répartition et d'usage de l'eau créés dans des bassins versants. En effet, de grandes seguias transféraient l'eau d'une rivière vers une autre ou même au-delà. Cependant, la carte de synthèse ignore les dimensions spatiales des usages des eaux. Elle reflète une pensée hydraulique limitée, vectorisée : les éléments représentés sont linéaires - les rivières et les canaux - ou ponctuels - les prises d'eau des canaux sur les rivières. Les convergences des eaux vers les bassins déversants, les zones réelles d'utilisation, sont esquissées mais en réalité pas documentées. Les périmètres d'utilisation de chaque canal sont ignorés. Comment dans ces conditions trouver un juste équilibre d'allocation à partir de quelques données ponctuelles recueillies de manière artisanale, dans un espace où les enjeux fonciers et hydriques reposaient sur des trajectoires historiques longues et des rapports de force conflictuels? 
Illustration 5 - Inventaire des canaux superficiels dans le Haouz de Marrakech, dans les années 1940

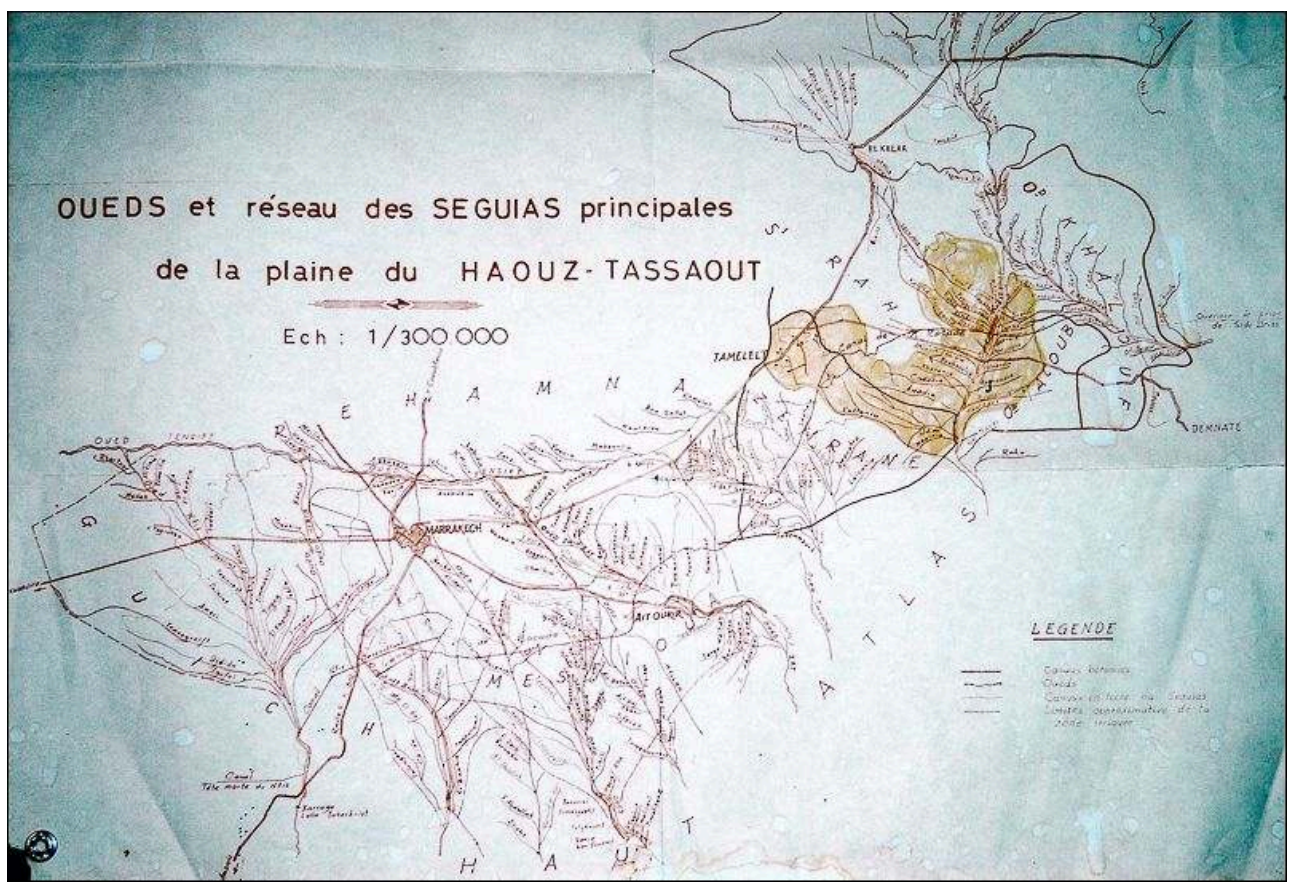

La zone orangée plus dense correspond à l'aménagement ancien de la Tessaout centrale, dans laquelle figure la Seguia Sultania, utilisée en particulier par les habitants de Tamelelt - voir illustration 13.

Source : Archives ORMVAH.

Toutes les procédures de reconnaissances des droits d'eau furent objet de contestations et de résistances dont on retrouve les témoignages dans les dossiers d'archives consultés à l'ORMVAH. Il faut souligner que l'irrégularité interannuelle du climat rendait parfois impossible la réalisation pratique des droits d'eau et donnait lieu à des altercations multiple, non seulement entre colons et indigènes, mais aussi entre colons eux même, avec parfois des conflits qui touchaient directement les intérêts des grands propriétaires terriens marocains. Au début des années 1930, le pouvoir colonial cherche à compenser le manque d'eau constaté autour de Marrakech, provoqué à la fois par des sécheresses mais aussi par la volonté d'octroyer de nouveaux droits d'eau aux personnes qui ne disposaient d'aucun accès sur les ressources déjà largement mobilisées par les systèmes anciens d'irrigation. Plusieurs idées furent avancées. La construction, dans les années 1930, du premier barrage réservoir sur l'oued Nfis appelé alors barrage Cavagnac (et par la suite Lalla Takerkoust) - entre dans cette stratégie, mais sa portée reste alors limitée. Les assemblées villageoises, les jmaâ, vont réagir vivement et exiger une amélioration de leurs propres accès à l'eau via les diverses seguias dont ils ont la charge. En fait, le grand projet colonial consiste à transférer les eaux du bassin de l'oued Lakhdar situé au Nord Est du Haouz jusqu'à l'oued Nfis par un grand canal dit de Rocade (Ducrocq, Zerhouni, 1987), un projet de très grande hydraulique qui ne se réalise pas avant l'indépendance du Maroc en 1956. 


\section{L'État, le privé et le communautaire de l'eau du Maroc indépendant}

19 Après son indépendance, le Maroc hérite de l'organisation administrative de l'ancien régime colonial. En matière de gestion de l'eau, la "mission hydraulique » est prolongée, amplifiée. Elle est conçue désormais à la fois comme un axe majeur du développement économique et une sorte de mobilisation politique et sociale. Le tournant intervient surtout dans les années 1970, avec le grand projet de la Banque Mondiale (Swearingen, 1987) qui permet au Roi Hassan II de mobiliser dans une contexte de crise sociale et politique le grand projet autour du slogan « un million d'hectares irrigués » (Ait Kadi, 1995 ; Attar et Ben Mohamed, 1987).

Pour autant, le Maroc ne connaît pas de ruptures brutales dans le monde rural mais plutôt une melkanisation ${ }^{12}$ des terres collectives. Entre 1955 et 1970, les exploitations agricoles des colons privées ou faisant partie des lots de colonisation, dont beaucoup disposaient de l'irrigation, connaissent deux issues : la cession volontaire par vente des domaines à de riches Marocains, et dans une moindre mesure l'expropriation engagée lors de la réforme agraire. De plus celle-ci est orientée pour une partie importante vers la création de grandes fermes d'État, administrées directement par des sociétés publiques. Parfois, sous l'effet des contestations émergentes dans les campagnes, les terrains expropriés sont re-cadastrés et exploités par le biais de coopératives très encadrées par le ministère de l'Agriculture. Les terres sont attribuées à des bénéficiaires, mais les cultures dirigées par des administrateurs, dans le cadre du modèle de Grande Hydraulique.

Après quelques années consacrées à l'état des lieux et des potentialités hydrauliques de chaque région, l'État conçoit un vaste plan d'investissements hydrauliques dans le cadre de l'office national d'irrigation, rapidement recomposé en neuf offices régionaux de mise en valeur agricole (ORMVA), avec des compétences très larges pour mettre en place de nouveaux réseaux d'irrigation le plus souvent sans aucun rapport avec les anciens dispositifs hydrauliques locaux. La gestion étatique suit toute la chaine de mobilisation, de transfert et d'utilisation des eaux, mais impose aussi un encadrement strict des filières de production et de commercialisation. Les nouveaux aménagements utilisent les dernières technologies hydrauliques disponibles avec l'appui de la coopération française et notamment des compagnies d'aménagement régional comme celle du Bas-Rhône-Languedoc (CNABRL) ou celle de la Société du Canal de Provence (SCP), créées juste après l'indépendance du Maroc en intégrant beaucoup des cadres hydrauliciens du protectorat. D'autres agences et bureaux d'études interviennent, en particulier d'Europe de l'Est, bulgares ou yougoslaves (El Faïz, 1999, 2001). Les ORMVA transforment radicalement les relations autour de l'eau, du foncier et de la planification des cultures et l'accès à l'eau suppose désormais le paiement d'une redevance (à l'exception des offices traitant des régions oasiennes).

L'objectif du million d'hectares est atteint autour de l'an 2000 (Debbarh et Badraoui, 2002). Dans ce calcul, on tient compte des très nombreux systèmes irrigués toujours gérés directement par les jmaâ hors des zones pilotées par les ORMVA (par exemple en zones de montagne ou dans les oasis). La PMH est simplement suivie et ses équipements éventuellement subventionnés, sans modification foncière et sans paiement de redevance. 

l'expérience de l'administration coloniale est utilisée dans la continuité avec les mêmes dispositifs de contrôle des aménagements hydrauliques locaux. Ainsi, dans cette région, le concept de Grande Hydraulique s'ajoute à l'expérience séculaire de la gestion de l'eau par différents types de personnes et d'institutions. Pour autant, l'office du Haouz engage en pratique la politique globale du pays et modifie l'organisation du partage de l'eau dans les différentes plaines de la région, de celle de la Tessaout au nord-est de Marrakech à celle du Nfis à l'ouest de la capitale régionale.

Dans les années 1960, l'ORMVAH conduit une étude très complète sur l'aménagement que les services hydrauliques du protectorat avait initié sans aller jusqu'au bout, en particulier le transfert d'eau du bassin de l'oued Lakhdar à celui du N'Fis. Ce projet fut repris dans les années 1970 en conservant cette fois le nom d'origine: le canal de Rocade détourne les eaux de l'oued Lakhdar et assure une alimentation nouvelle pour de nombreux secteurs le long des 120 kilomètres de son parcours (illustration 6). Cependant, la jonction avec l'oued Nfis n'est pas réalisée, car entretemps, la question de la réorganisation des réseaux s'est posée entre ce qui existait déjà à partir du barrage Lalla Takerkoust et ce qui se met en place dans les années 1980 avec la nouvelle eau de Rocade. Ainsi, au lieu d'alimenter en eaux supplémentaires les anciennes seguias, il a été décidé de construire un nouveau réseau de tuyaux souterrains selon une configuration totalement nouvelle et transversale aux anciens réseaux superficiels. L'eau du barrage de Lalla Takerkoust arrive à un bassin intermédiaire puis circule dans le système de tuyaux avec la pression naturelle créée par le dénivelé entre le bassin et la zone irriguée. De la même manière, l'eau de Rocade débouche sur un autre bassin situé à proximité de l'oued Ba'ja, et sa mise en pression se fait dans un autre système de tuyaux desservant une autre zone irriguée. Cependant, les anciennes seguias n'ont pas été abandonnées. En effet, malgré la présence du barrage, il y a encore de l'eau dans l'oued N'fis, relativement rare au niveau des résurgences, mais parfois très abondante en période de crues en provenance des affluents non régulés, voire du barrage luimême lorsque les fortes pluies tombent au moment où il est déjà rempli au maximum. En fait, cette organisation hydraulique complexe existe dans presque tout le Haouz. On peut parler de coexistence de réseaux d'époques différentes, aussi bien superficiels que souterrains. Si les khettaras et les puits traditionnels ont coexisté plusieurs décennies avec les forages et les stations de pompage, et n'ont pu que cesser de fonctionner du fait de l'extraction trop forte de ces formes modernes d'accès à l'eau, en revanche les seguias en terre continuent de servir en dépit de l'installation des réseaux modernes de l'ORMVAH.

Quoi qu'il en soit, le transfert d'eau de l'oued Lakhdar vers Marrakech satisfait en partie ceux qui ont pu obtenir un nouvel accès à l'eau, mais pénalise fortement les utilisateurs en aval de la prise du canal de Rocade. En effet des dizaines de seguias recevaient auparavant l'eau de l'oued Lakhdar. Certaines zones de la rive gauche ont pu être connectées au nouvel aménagement créé par l'ORMVAH dans ce que l'on appelle alors la Tessaout centrale, mais d'autres n'ont pas obtenu de compensation hydrique ${ }^{13}$. L'oued Lakhdar est en fait un affluent de l'oued Tessaout, lui-même profondément remanié dans sa gestion par la construction d'un autre barrage réservoir, le barrage Moulay Youssef. Plus en aval encore, les ingénieurs nationaux établissent un autre canal de transfert appelé « T2 " pour apporter à la «Tessaout Aval» une dotation en 
eau issue du barrage de Ben El Ouidane, en quantité limitée, puisqu'elle ne représente que la moitié des apports existant avant la mise en place du canal de Rocade.

Illustration 6 - Carte de l'aménagement de l'ORMVAH dans les années 1980-1990

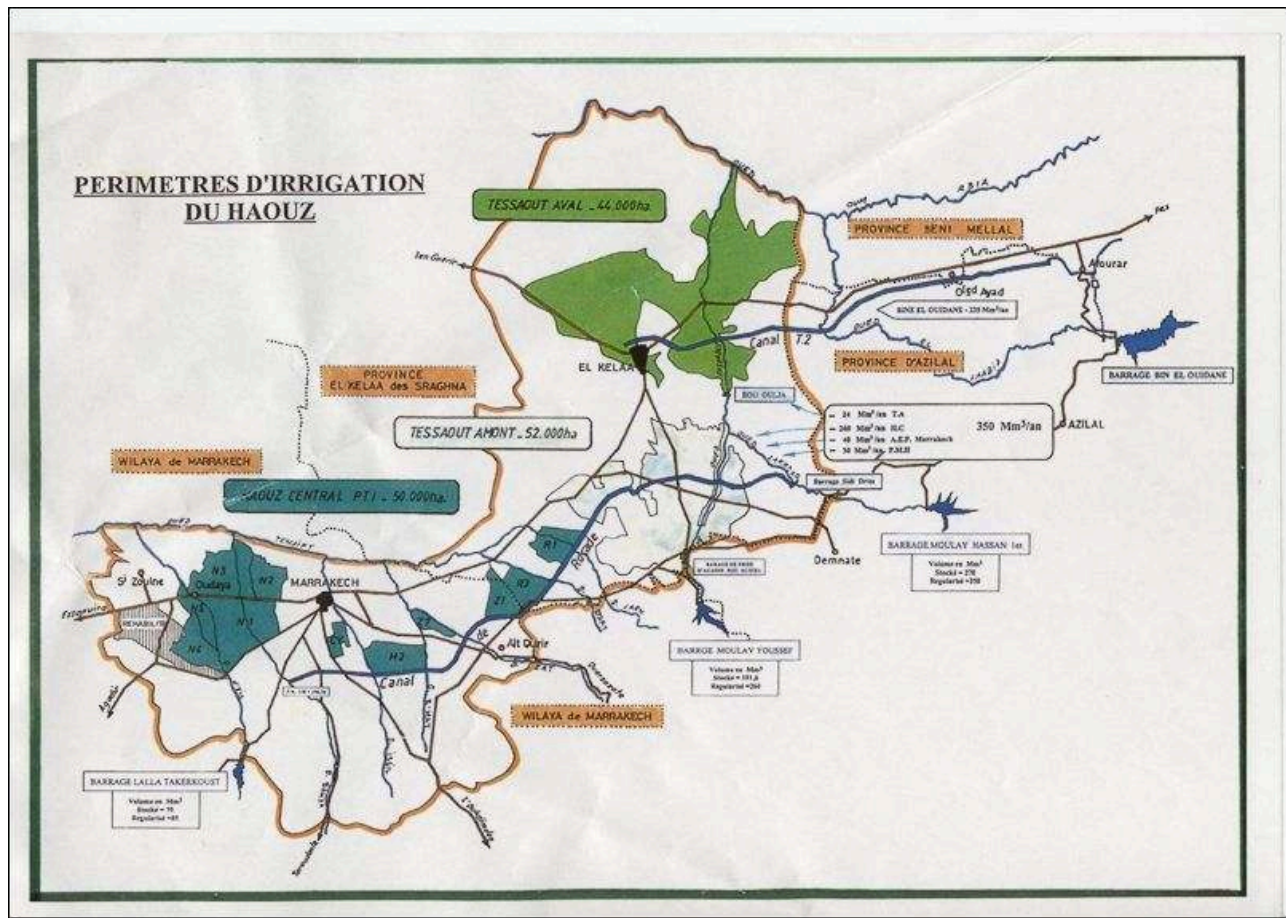

Les anciens aménagements associés aux différents cours d'eau descendant du Haut Atlas disparaissent de la vision des ingénieurs, alors que la plupart des seguias existent toujours comme le décrit l'illustration 5

Source : Archives ORMVAH.

Au cours des trente dernières années, la vision de l'office du Haouz a complètement changé. À sa création au milieu des années 1960 et dans la première décennie de son fonctionnement, l'office est une sorte de grande agence régionale de régulation des ressources en eau qui sont générées dans les hautes vallées et descendent vers la plaine du Haouz via les oueds, les dérivations des seguias et la dynamique des nappes d'eau souterraines. L'Office tient compte de toute la complexité du réseau de seguias et de khettaras. Mais après la construction de trois barrages et la mise en place des canaux de transfert, il semble que la gestion locale de l'eau de chaque oued soit oubliée. Les secteurs hydrauliques anciens semblent méconnus, en tous cas marginaux, du point de vue de leur fonctionnement hydraulique et agricole mais aussi du point de vue de leurs formes institutionnelles toujours fondées sur des notions de bien commun, de partage et d'assemblée, les jmaâ. Les systèmes de mesure et de surveillance sont simplifiés ou disparaissent, et finalement les fonctionnaires de l'office travaillent principalement au plan de gestion intégré des seuls nouveaux réseaux. De fait, le canal de Rocade est équipé du très sophistiqué système de régulation dynamique mis au point par la Société du Canal de Provence.

En réalité, l'intégration technologique bute sur de nombreux problèmes. Les ressources en eau diminuent du fait de sécheresses plus intenses dans les 20 dernières années $d u$ $\mathrm{XX}^{\mathrm{e}}$ siècle. Les autorités politiques ont choisi de fournir de l'eau à de nouveaux secteurs tout au long du transfert. Il n'est plus possible de faire fonctionner l'ouvrage selon la 
demande des irrigants bénéficiaires. L'ORMVAH institue un quota d'eau très bas, fixé à 6000 mètres cubes par an et par hectare, alors que l'évapotranspiration potentielle est trois fois plus forte, 15000 mètres cubes par hectare et par an (Simmoneaux et al., 2009).

Nous avons trouvé dans les archives de l'office du Haouz une carte de grand format réalisée en 1964 qui détaille le plan de modernisation envisagé à l'époque (illustration 7). Ce document donne une idée assez précise de ce qui va être réalisé dans les trois secteurs du Haouz central, autour de Marrakech, de la Tessaout centrale et de la Tessaout aval à l'Est. Ce qui est plus original dans ce document est qu'à côté des détails de la sectorisation des périmètres modernisés de la plaine, figure également la représentation détaillée des hauts bassins versants montagnards.

Illustration 7 - Carte du nouvel aménagement hydraulique du canal de Rocade réalisé dans les années 1960

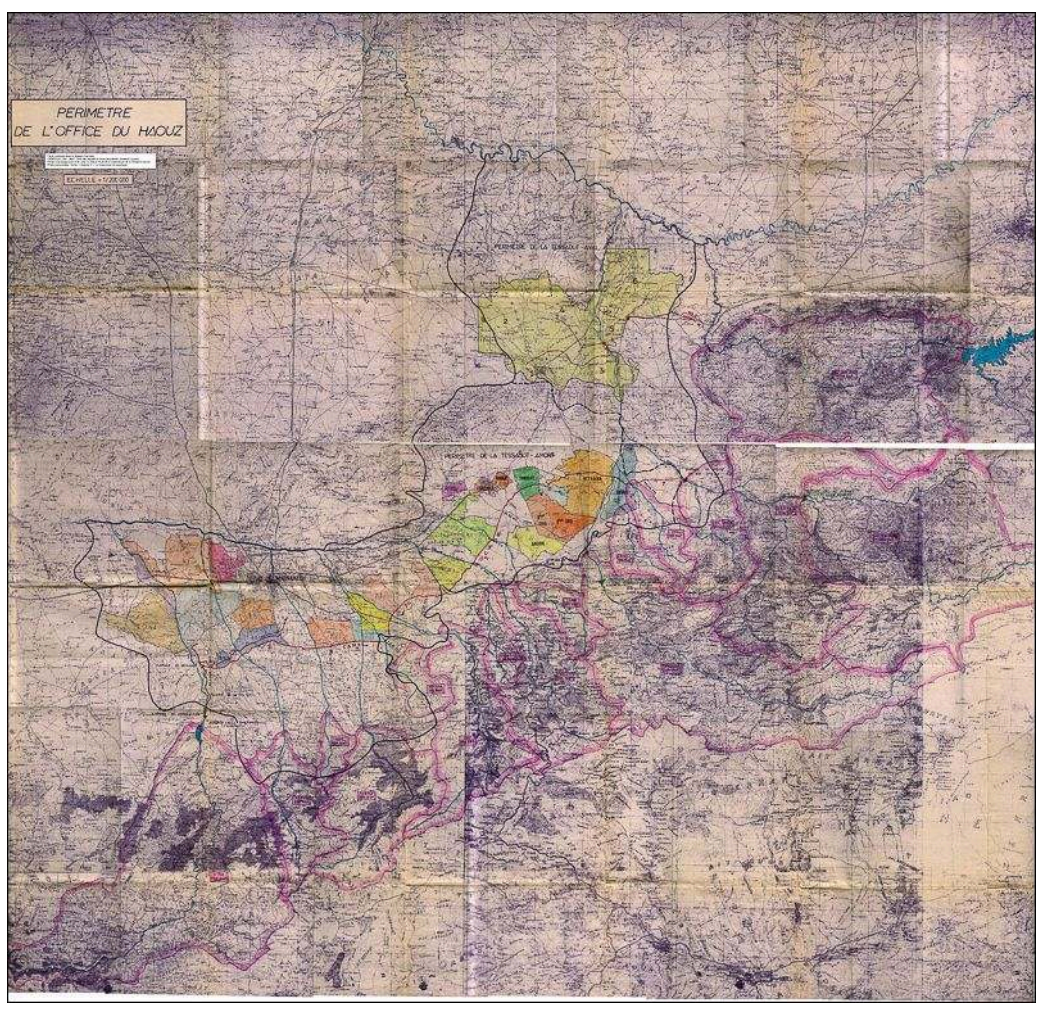

Archives ORMVAH, 1964.

Afin de comprendre l'information très utile de la territorialisation des eaux du Haouz, nous avons scanné cette carte de 1964 puis traité les informations contenues en les séparant par calques, comme par exemple l'hydrographie, les contours de bassins, les périmètres projetés. Sur cette base, nous pouvons recomposer le document sous forme plus dynamique, en proposant une sorte d'animation cartographique pédagogique lors de projection sur un écran en salle (figures 7a à 7d). En effet, plusieurs interprétations de la carte sont possibles et correspondent aux différentes visions du territoire du Haouz, dont la délimitation a toujours été objet de controverses (Pascon, 1983), en lien avec les disciplines des observateurs. Les gestionnaires de l'eau de l'ORMVAH y voient une grande unité de planification administrative de travaux hydrauliques dans la plaine: c'est le domaine d'action de l'ORMVAH (illustration 7a). Les géographes et 
hydrologues insistent sur les composantes hydrographiques et identifient 16 bassins versants montagnards fournissant diverses eaux à la plaine, et seulement trois de ces bassins auront une régulation des débits par un barrage réservoir (illustration $7 \mathrm{~b}$ ). Les historiens s'attachent aux structures hydrauliques anciennes qui dérivent les eaux des oueds non régulés, et se perpétuent aussi sur les oueds régulés (illustration 7c). Enfin, les économistes ne perçoivent plus que le grand canal de Rocade apportant un service moderne de distribution d'eau payant à une clientèle particulière (illustration $7 \mathrm{~d}$ ).

Illustration 7a - La zone d'action de l'ORMVAH : une plaine aride au nord des montagnes du Haut Atlas

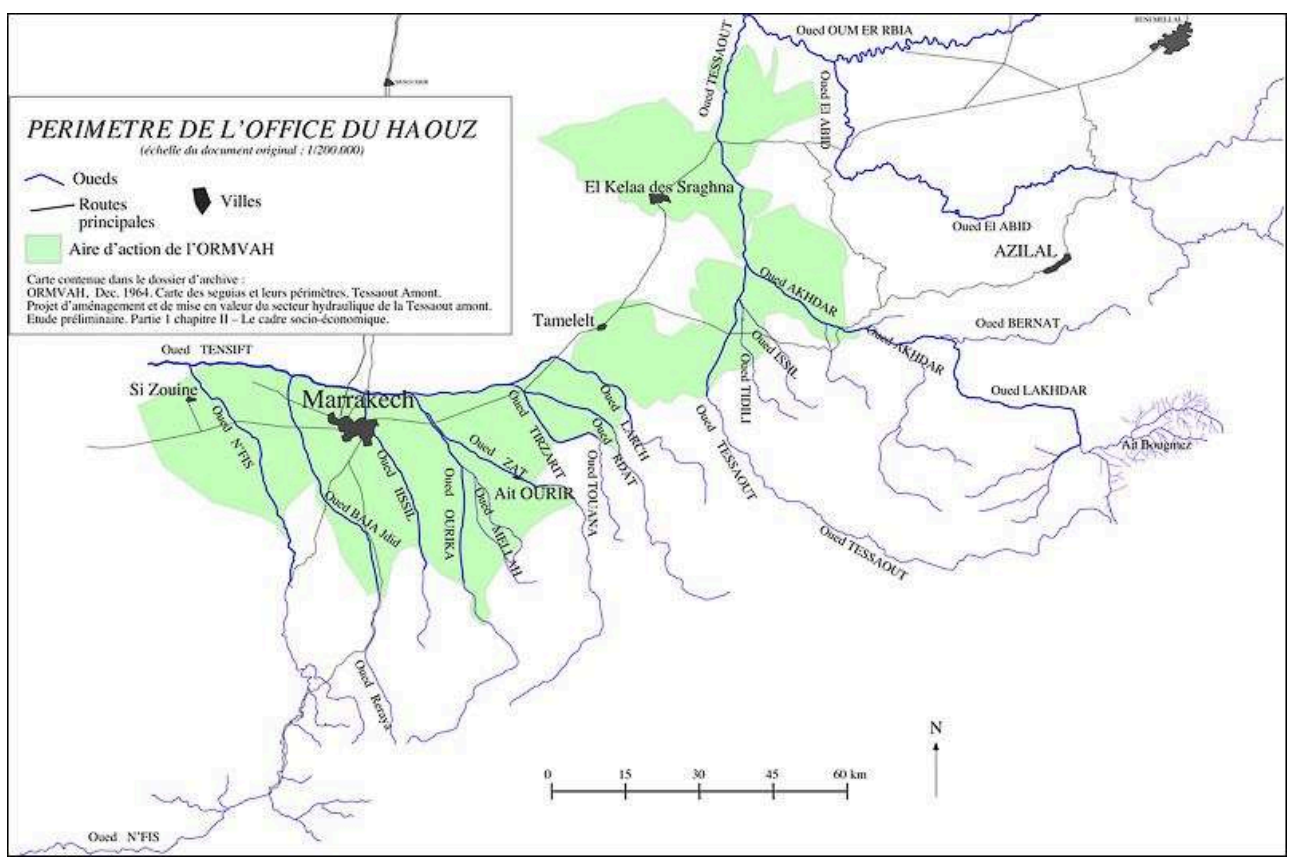


Illustration $7 \mathrm{~b}$ - La complexité des bassins versants montagnards alimentant les 12 principales rivières de la plaine

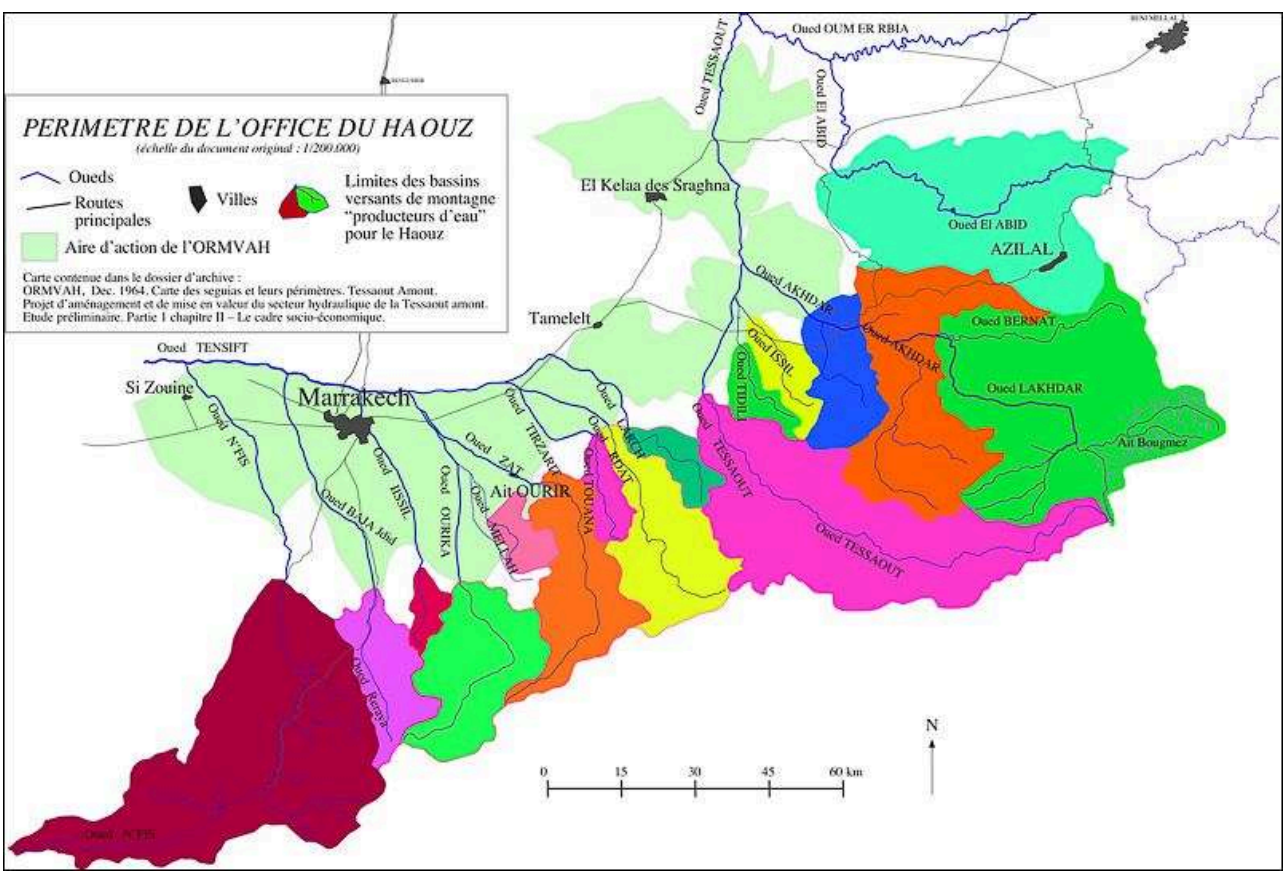

Illustration 7c - La représentation des centaines d'anciens canaux de surface, seguias, répartissant les eaux venant de la montagne et des bassins supérieurs

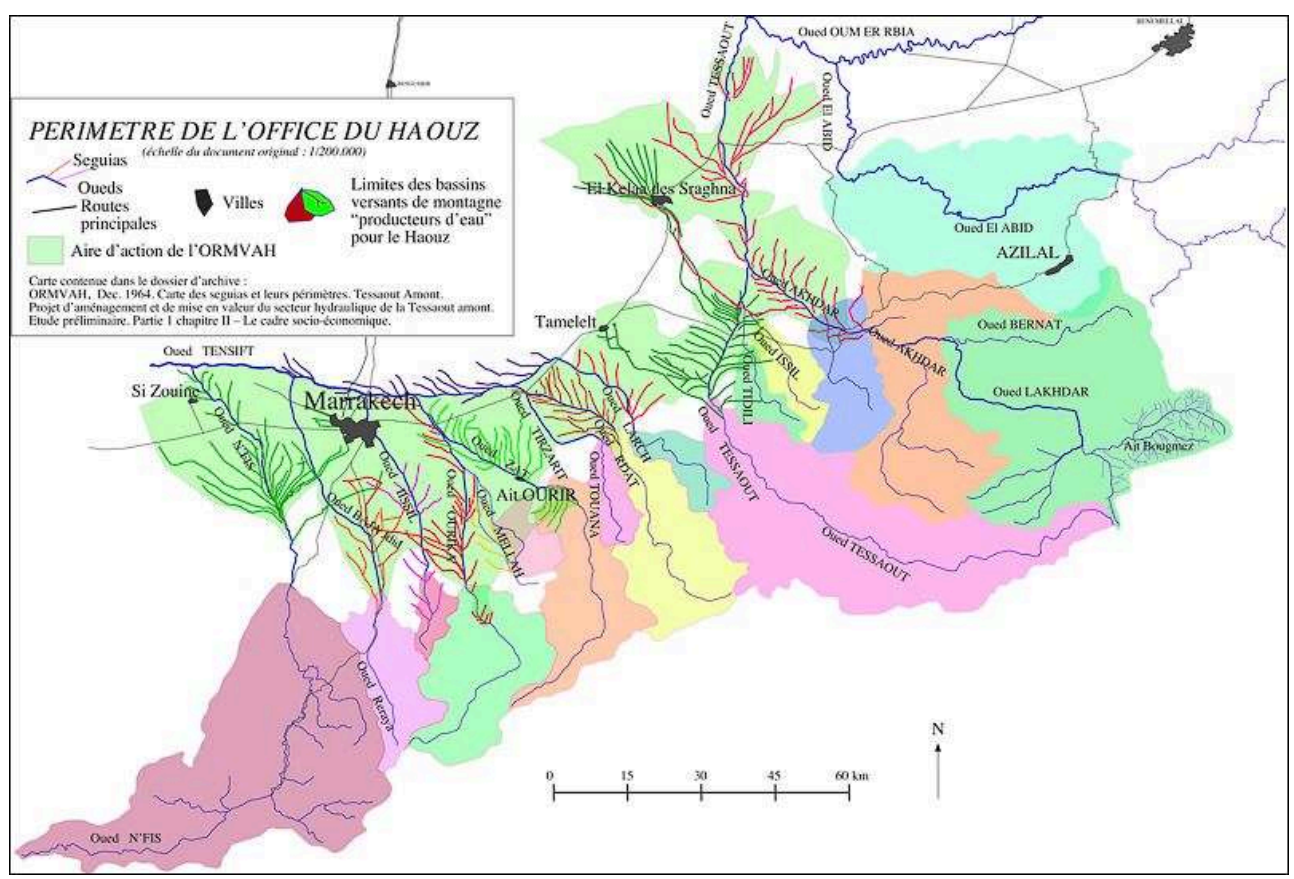


Illustration $7 \mathrm{~d}$ - La vision nouvelle et simplifiée de la bureaucratie hydraulique à la fin du XXe siècle

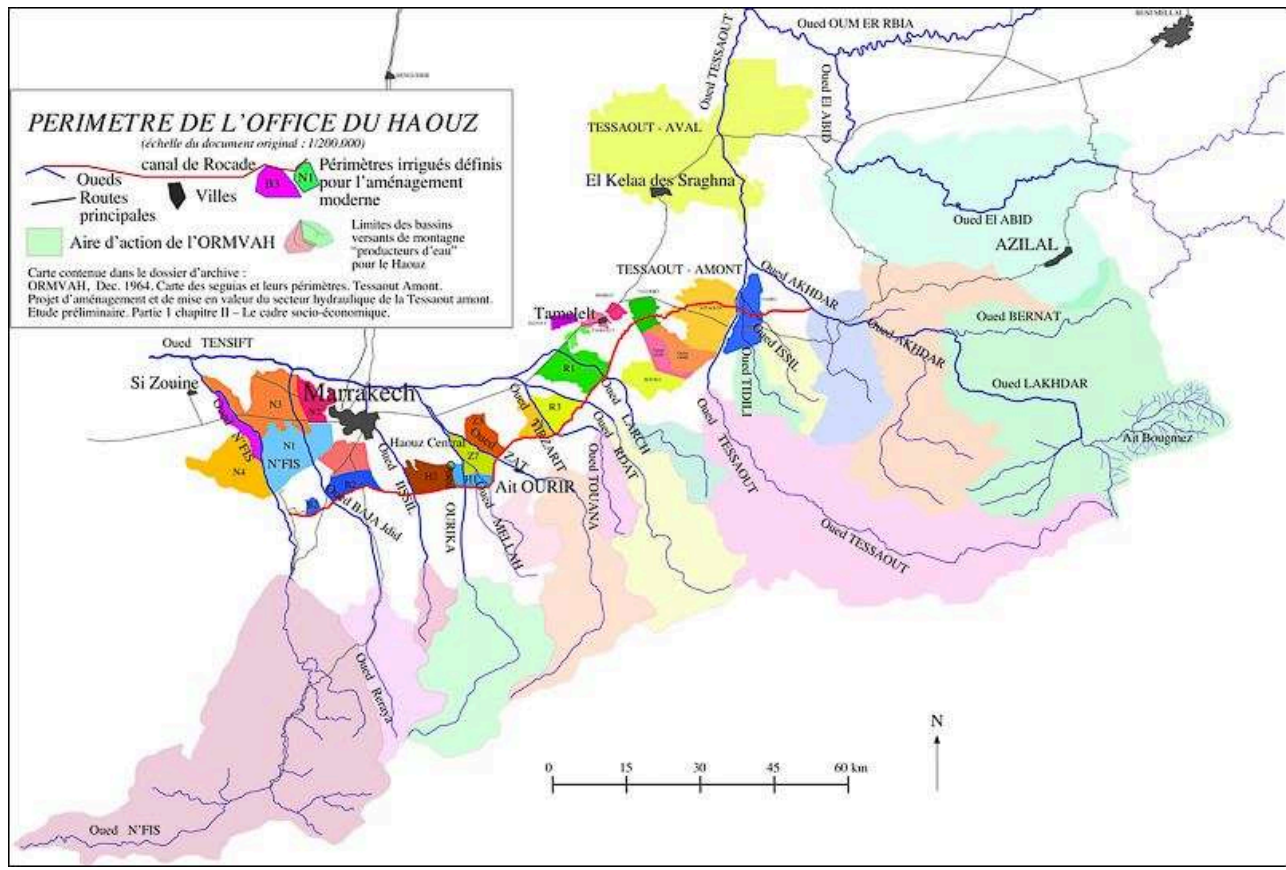

Nous pourrons ajouter par la suite de nouvelles couches d'information issues d'autres auteurs de représentations du territoire comme la Direction provinciale de l'Agriculture qui s'intéresse à l'ensemble des seguias de montagne et à certaines seguias de la plaine, ou comme l'Agence de bassin du Tensift, plus récemment apparue comme entité régulatrice d'une partie du Haouz, et dont le travail porte surtout sur la surexploitation de l'eau souterraine. Mais à ce stade, nous préférons nous référer à des études plus précises pour comparer les formes locales de gestion des différentes eaux et les liens qui s'établissent entre tous les auteurs de représentations.

\section{De la vision globale à l'éclairage des situations locales : trois exemples d'incompréhensions entre parties prenantes de la gestion de l'eau}

La diversité des délimitations des zones de gestion des eaux le laisse entrevoir : les modèles d'intervention ne sont pas identiques dans tous les secteurs de la zone d'action de l'office du Haouz. Les distinctions portent d'abord sur l'histoire ancienne des aménagements, précoloniale et coloniale. Elles jouent aussi sur les 50 ans qui suivent l'indépendance. Les concepts de développement de la grande hydraulique évoluent aussi sous l'effet des technologies en vogue (reprise d'anciens canaux, création de canaux en béton portés, création de réseaux de tuyaux sous pression). L'expérimentation successive de projets de reconfiguration autoritaire des réseaux fournit divers bilans et ajustements. Le premier modèle est très lié au processus même de décolonisation, avec l'expropriation de fermes européennes et la constitution de fermes d'État dépendantes du Ministère de l'Agriculture. Il se conjugue en partie avec la marocanisation ${ }^{14}$ de l'administration hydraulique et de l'autoritarisme en matière de construction de barrages et de transfert inter - bassins. Le deuxième modèle est l'application d'une triple révolution hydraulique, foncière et agricole mise en œuvre 
dans divers offices régionaux de mise en valeur agricole comme le Gharb ou le Doukala, et également mise en application dans le courant des années 1970 dans le Haouz, pour le secteur de la Tessaout centrale situé à une centaine de kilomètres au nord-est de Marrakech.

Là, le canevas hydraulique est entièrement redessiné. Les nouveaux canaux ne sont plus alimentés par des prises en rivière mais par un dispositif technique employant un tunnel et des canaux en béton portés, dominant les secteurs d'arrosage. La trame foncière ancienne disparaît du fait de l'expropriation ou de la reprise en main par l'État de terres collectives. La nouvelle trame foncière qui en découle reprend les délimitations géométriques du réseau de canaux secondaires, tertiaires et quaternaires, dont la dénomination officielle est "la trame B ", en opposition avec la "trame A » quand les structures foncières ne sont pas remises en cause (Kleiche-Dray, 2001). Les attributions foncières de la «trame $\mathrm{B}$ » prévoient une répartition des lots cultivés en fonction de grandes soles techniques, objet de la planification des cultures. Les attributaires sont aussi tenus de s'inscrire dans ce plan et d'alimenter les filières organisées par l'État. Selon les secteurs réaménagés, des soles sont réservées pour le coton, pour les agrumes, et parfois pour les fourrages en vue de favoriser l'élevage. Les attributaires doivent suivre les décisions du Ministère de l'Agriculture et de l'office et payer une redevance pour l'eau, après un différé plus ou moins long. Dans les années 1990, d'autres modèles d'aménagement suivent, plutôt portés sur le paiement de services de distribution de l'eau par des individus ou des groupements d'individus, sans obligation de culture. On aboutit à un ensemble finalement assez hétérogène lié en grande partie au modèle de mise en valeur imposé à chaque époque et aux formes de désengagement après la réforme agraire ou après le recul de la planification des cultures.

Le bilan macroéconomique de la Grande Hydraulique amène les bailleurs de fonds à s'interroger sur le modèle privilégié d'autoritarisme hydro-agricole. Sous influence directe de l'école des commons d'Elinor Ostrom, la Banque mondiale conditionne la poursuite des prêts consentis à l'hydraulique agricole par la promotion de la gestion participative de l'irrigation. Dans la Tessaout, comme dans les autres zones de mise en valeur de l'ORMVAH, sur ces secteurs hydrauliques finalement assez hétérogènes dans leurs obligations, arrive l'idée de la participation des irrigants à la gestion de l'irrigation dans des structures formelles, les associations d'usagers de l'eau agricole (AUEA), une idée ancienne que le protectorat avait aussi expérimenté mais uniquement dans les périmètres de colonisation avec la notion d'association syndicale d'agriculteurs privilégiés (ASAP). Après l'indépendance, et malgré les interrogations de Paul Pascon dont nous avons rendu compte plus haut, la reconnaissance d'institutions locales de gestion de l'eau n'avait jamais été envisagée, ni en PMH, ni a fortiori en Grande Hydraulique. Tandis que ces nouvelles configurations d'AUEA se territorialisent, le plus souvent à l'initiative des offices, le recours à des pratiques d'accaparement des eaux souterraines par des individus capables de maîtriser les technologie de forage et d'investir dans l'équipement de pompage, entraîne une dispersion très forte des points de captation des eaux souterraines, un rabattement très rapide des nappes surexploitées et rend la régulation et le contrôle de la distribution de l'eau de plus en plus difficile. 


\section{Le Haouz Central : une mosaïque de différentes expériences d'irrigation}

Revenons aux archives de l'ORMVAH et au travail de Paul Pascon (1983). Il rassemble alors les informations sur toutes les techniques d'irrigation et les modes de gestion de l'eau dans le Haouz central. Il décrit les différents systèmes de galeries prélevant des eaux souterraines (khettaras), les systèmes de canaux dérivant et partageant les eaux superficielles (seguias) et les systèmes exploitant aussi les nappes phréatiques à partir de puits et de pompes. Il explique que dans la plupart des lieux, ces systèmes sont combinés. Finalement, l'arrivée du Canal de Rocade dans le Haouz central s'opère dans des zones déjà aménagées, avec un paysage humanisé structuré par de grandes et de petites exploitations qui sont liées à différentes communautés gestionnaires d'eau superficielle ou souterraine, avec leurs institutions propres (illustration 8a).

Illustration 8a - Carte des secteurs hydrauliques du Haouz Central en 1976

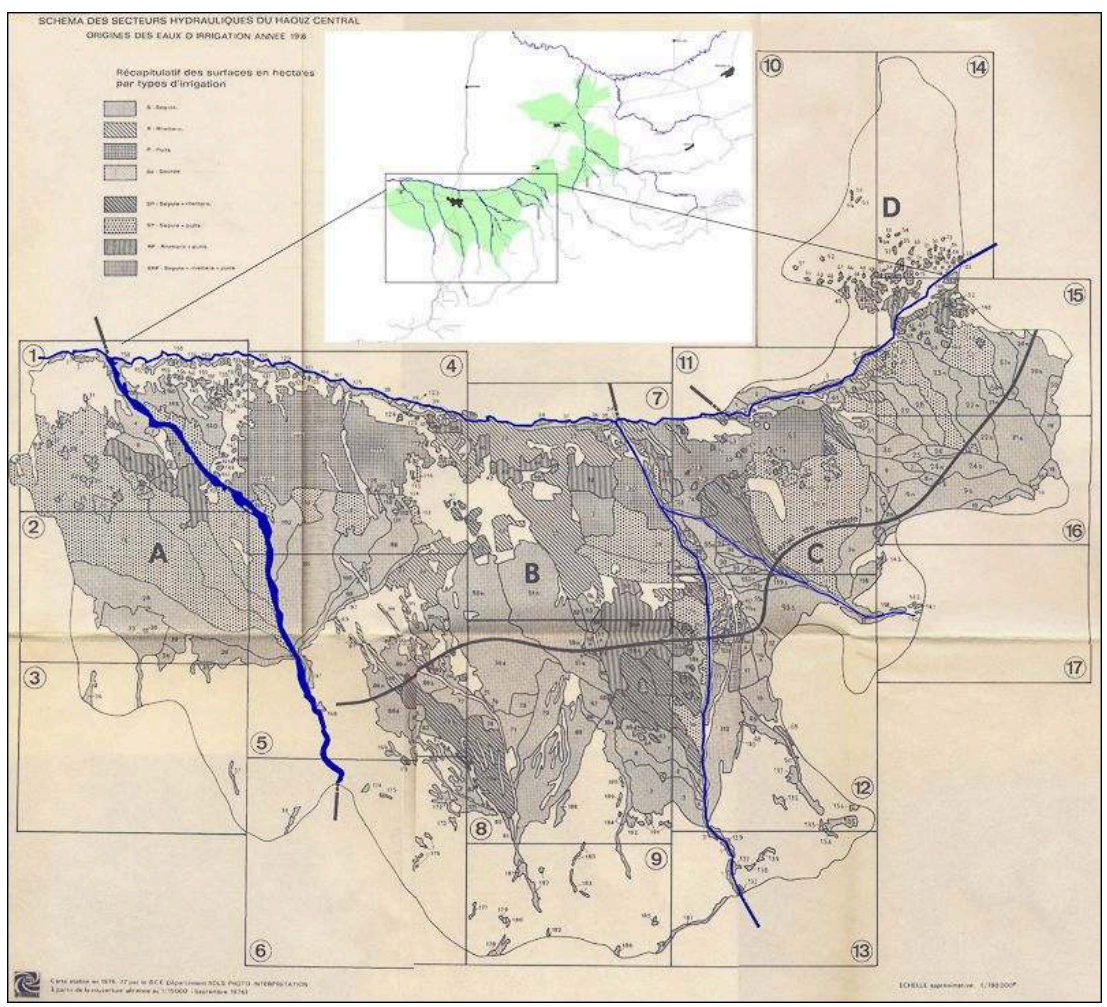

BCE Département sols. Photo-interprétation à partir de la couverture aérienne au 1/15.000, septembre 1976.

Source : Archives ORMVAH, Marrakech.

Pour bien saisir la coexistence et les combinaisons des différents réseaux historiques encore en place, nous recomposons la carte originale en couches d'informations pour retrouver les zones connectées à chaque type de réseaux. À l'intérieur de l'aire irriguée générale (illustration $8 \mathrm{~b}$ ), on repère clairement les trois grands types de dispositifs d'arrosage qui se recouvrent en de nombreux lieux, l'aire alimentée par les khettaras, l'aire reliée aux seguias et celle dépendant de pompage motorisé. 
Illustration 8b - Cartes des aires connectées aux différents types de ressources en eau

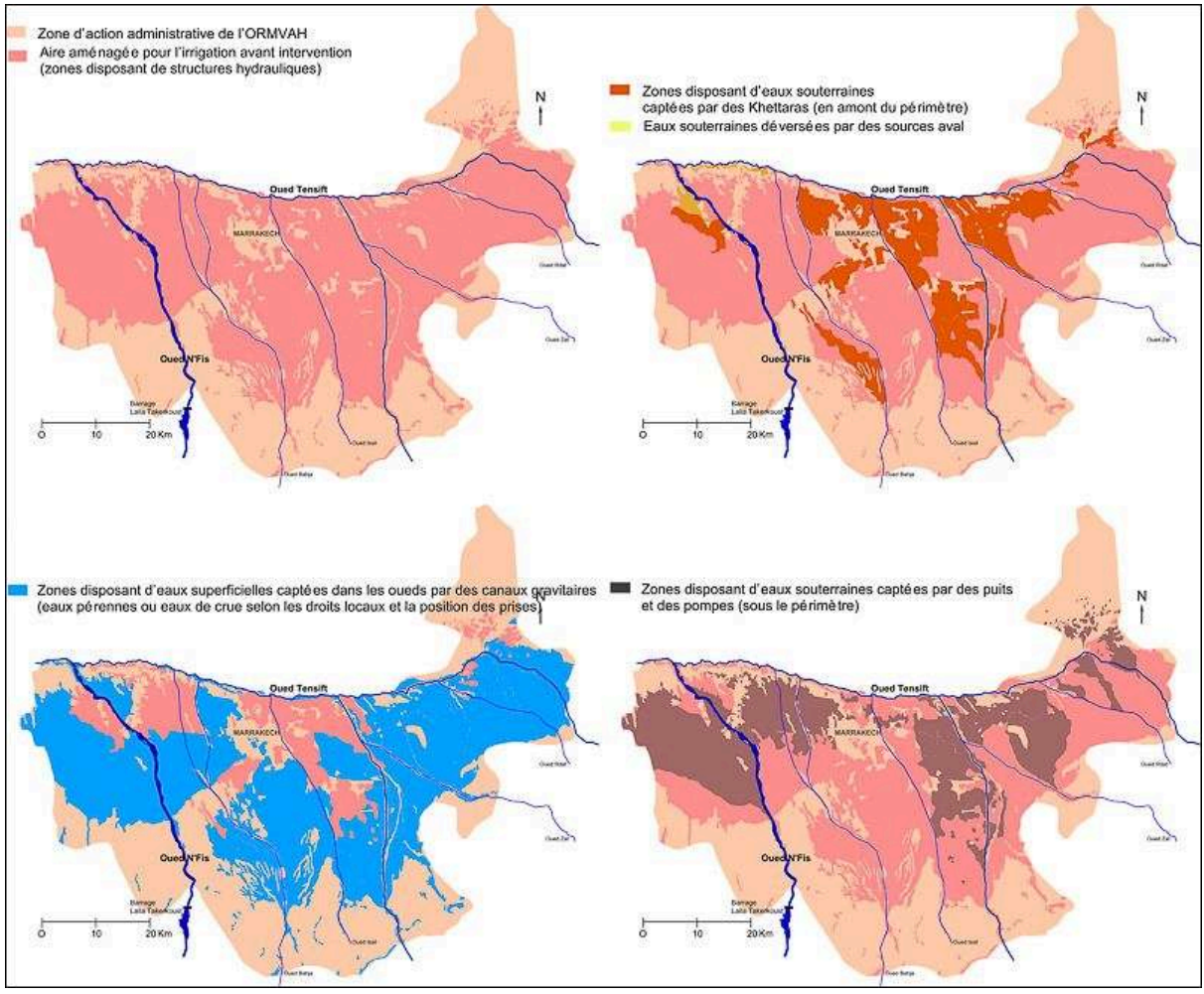

La superposition des trois types d'aires connectées aux trois types de réseau se traduit par une mosaïque de territoires hydrauliques (illustration 8c). Le canal de Rocade est une sorte de greffe d'un nouvel apport d'eau, un quatrième dispositif associé ou non aux précédents, de façon inégale et singulière. Même si elle est intégrée dans une zone donnée du Haouz central, elle n'est pas gérée par les institutions qui s'occupent déjà des autres réseaux. L'eau du canal de Rocade est administrée directement par l'office dont les dirigeants et les cadres ignorent ou minimisent l'importance des formes locales de gestion des eaux. Finalement, c'est une vision simplificatrice qui surgit avec une attention portée aux seuls espaces connectés au canal de Rocade, malgré l'existence des autres réseaux qui fonctionnent encore, même si celui des khettaras connaît une crise majeure à partir des années 1970-1980. 


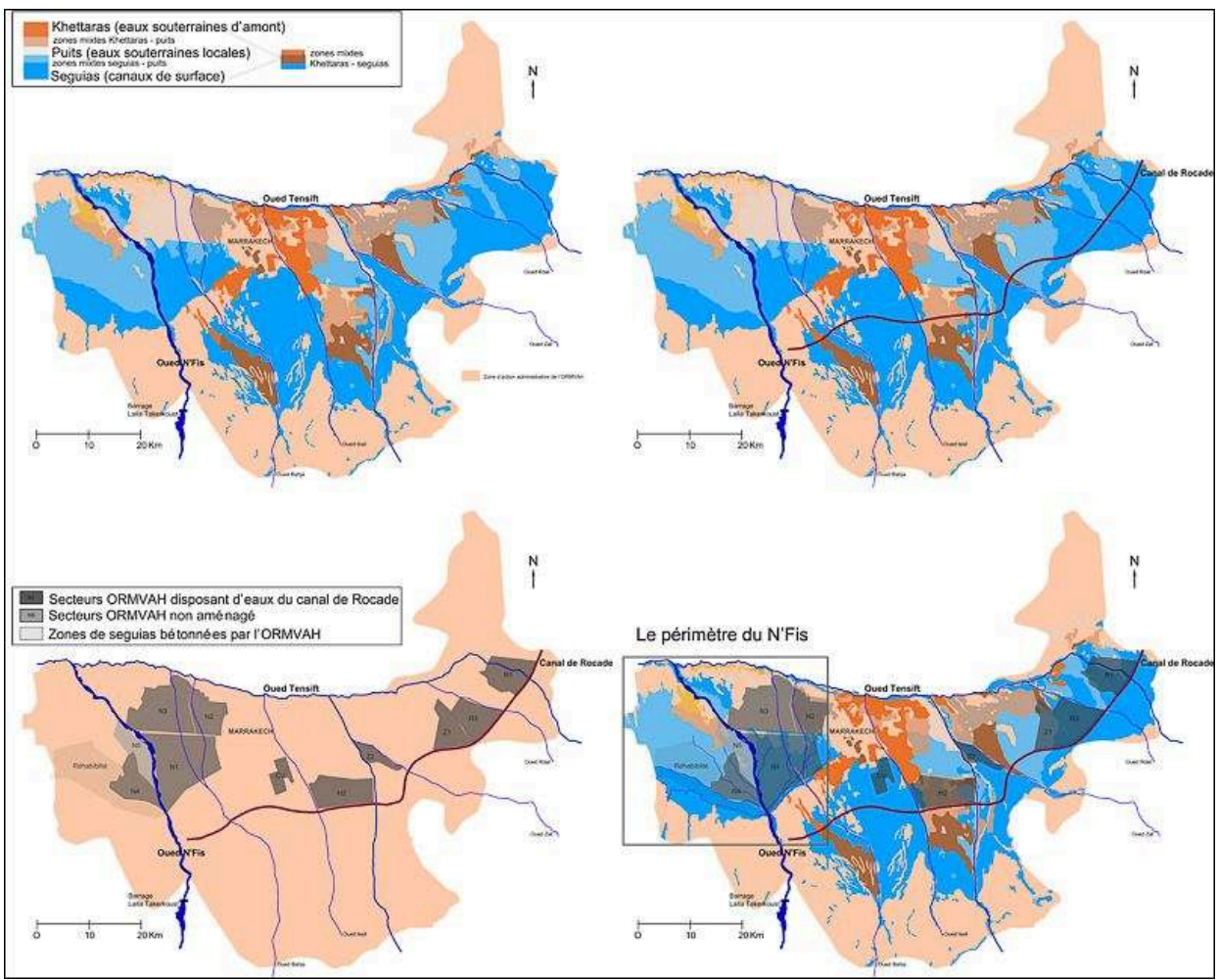

Recomposition de la carte en partant de la mosaïque des systèmes anciens avant l'arrivée du canal de Rocade, la mise en place du canal et la focalisation de l'office du Haouz sur les périmètres desservis par le canal de Rocade.

\section{La Tessaout centrale : une expérience radicale de réaménagement hydraulique et foncière}

Le projet de modernisation de l'irrigation du Haouz central vient chronologiquement après la premier projet de réorganisation de l'hydraulique agricole de la Tessaout, une zone située au nord-est de Marrakech. Là, a été imaginé et mis en œuvre une planification intégrée en rupture complète avec les anciens modes de gestion de l'eau, selon des principes assez proches du despotisme hydraulique proposé par Wittfogel (1957). Pour beaucoup de cadres marocains et internationaux, venant d'horizons variés d'Europe orientale et occidentale, la manière forte était considérée comme une étape nécessaire dans le développement des infrastructures.

L'oued Tessaout est aussi un site très connu pour la longue histoire des réseaux d'irrigation de surface, avec des canaux, les seguias, disposés de part et d'autre du lit principal (illustration 9). Du point où la Tessaout quitte la haute montagne, au point médian dans la vallée où se produit la jonction avec l'oued Lakhdar, on dénombre 21 seguias et plusieurs centaines de branches de distribution, les mesref, qui fournissaient jusque dans les années 1970 des parts d'eau à des groupes spécifiques des communautés d'usagers. La disposition géographique géométrique de ces composantes a été discutée par divers auteurs marocains (Herzenni, 1975 ; Pascon, 1983). Elle reste encore insuffisamment expliquée. L'eau est distribuée dans chaque village sans qu'il n'y ait de priorité de l'amont sur l'aval. Il existe des formes d'accès à l'eau pour chaque village, dont le terroir irrigué comprend une périphérie proche des habitations 
dénommée justement "haouz du douar ", littéralement la banlieue du village. Cette zone généralement constituée d'une oliveraie en partie cultivée sous les arbres, reçoit en principe l'eau permanente disponible, tandis que la zone de plein champ au-delà du Haouz du Douar ne reçoit de l'eau que lors des épisodes de crue.

Illustration 9 - Carte de situation des zones étudiées

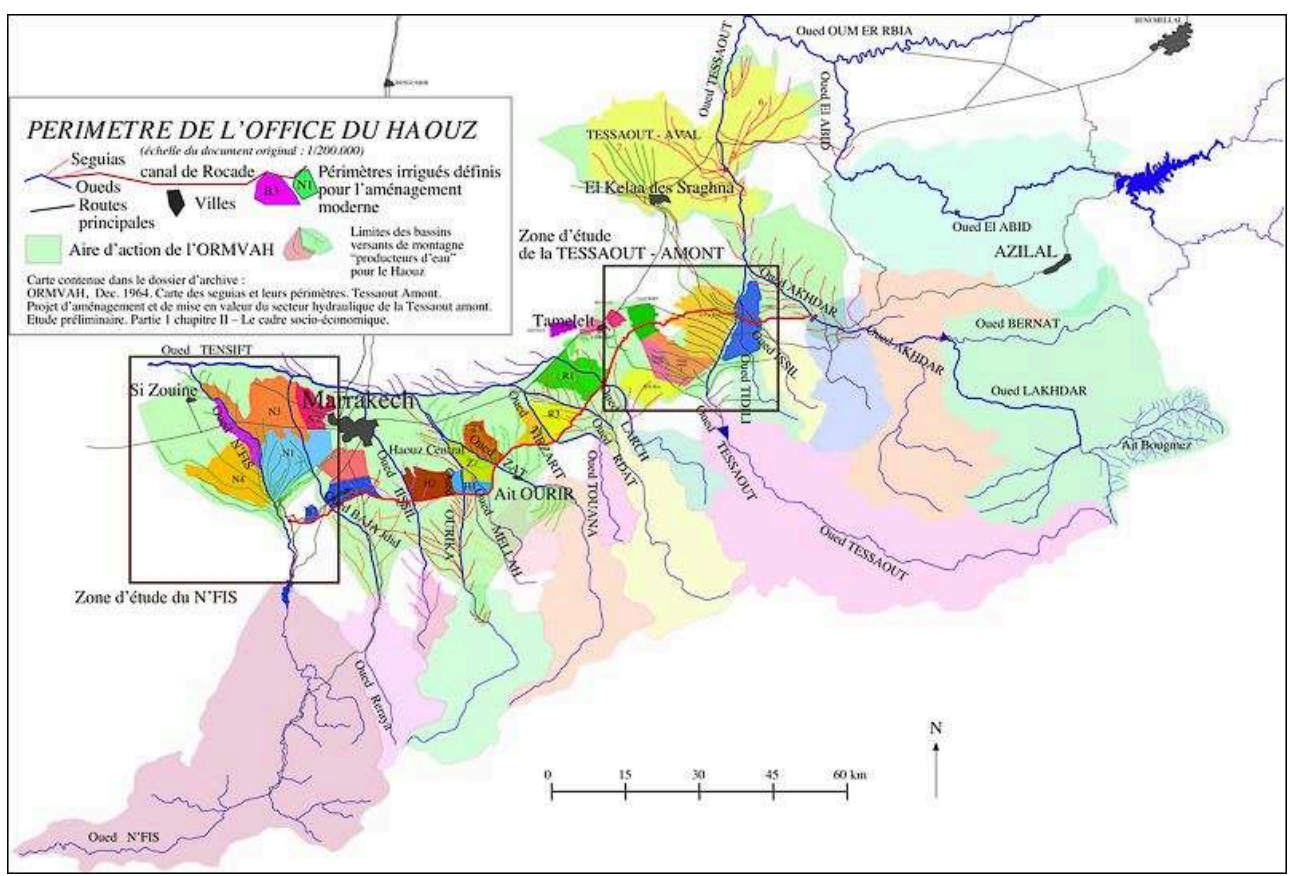

On retrouve la Tessaout à l'est, le N'Fis à l'ouest de Marrakech.

La Tessaout a été le théâtre de la première expérience de réaménagement hydraulique dans la région du Haouz. Elle s'est faite en rupture avec le canevas hérité de la région. Après la construction du barrage Moulay Youssef en 1969 sur l'oued Tessaout, les nouvelles autorités hydrauliques ont décidé de détruire l'ensemble des prises d'eau et canaux en terre et de les remplacer par un réseau indépendant, en béton et souvent semi-porté (illustration 10). Certes, les canaux en demi-lune suivent plus ou moins le trajet des anciennes seguias dans le nord de la plaine irriguée mais dans le sud et l'ouest, les nouveaux réseaux n'ont plus rien de commun avec les anciens. 
Illustration 10a - Carte de l'aménagement ancien de la seguia Sultania à la jonction avec la rivière Lakhdar

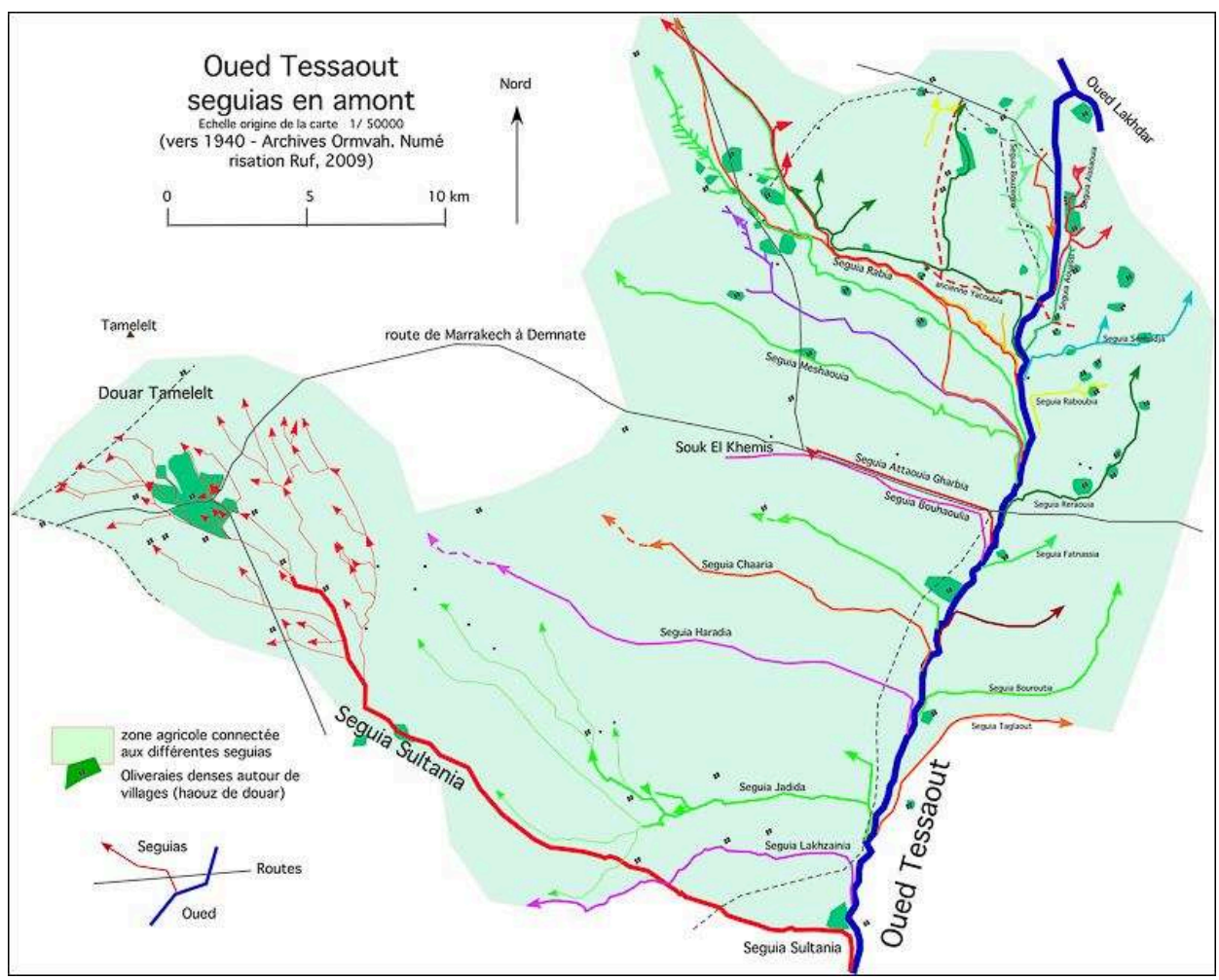

Ce secteur était dénommé Tessaout centrale dans les années 1960-1970. 
Illustration 10b - Carte de la Tessaout amont dans les années 1970 avec le nouvel aménagement

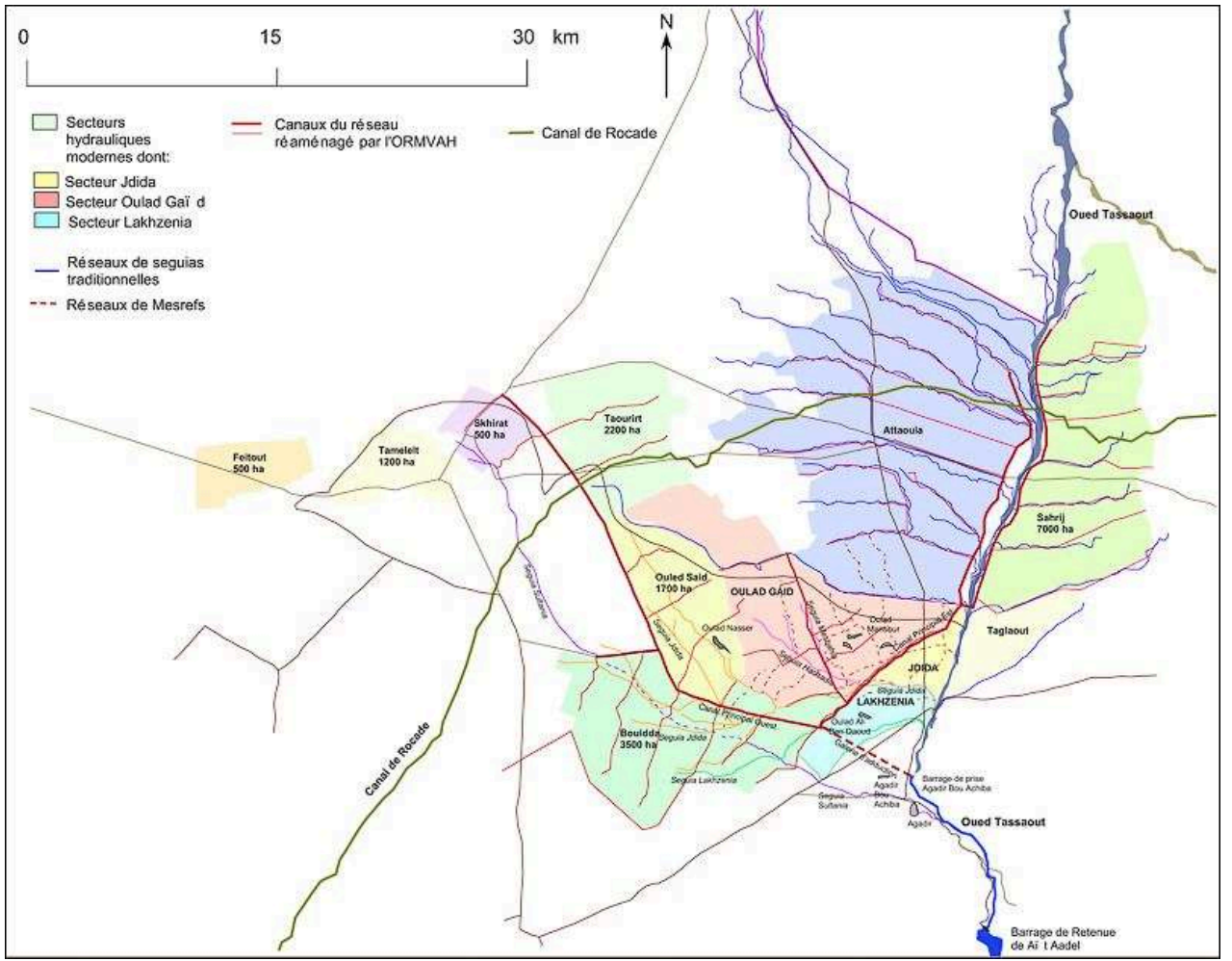

Les prises d'eau des anciens canaux ont été détruites. Les terres ont été redistribuées selon le modèle de planification foncière et hydraulique de la « trame B », comme cela apparaît sur l'image satellite (sources : google earth, 2009). La Seguia Sultania, à l'amont, reste en place pour desservir les personnes qui ne pouvaient être intégrées au nouveau système. Elle existe toujours pour desservir à l'aval les coopératives et fermes d'État dans la zone de Tamelelt. 


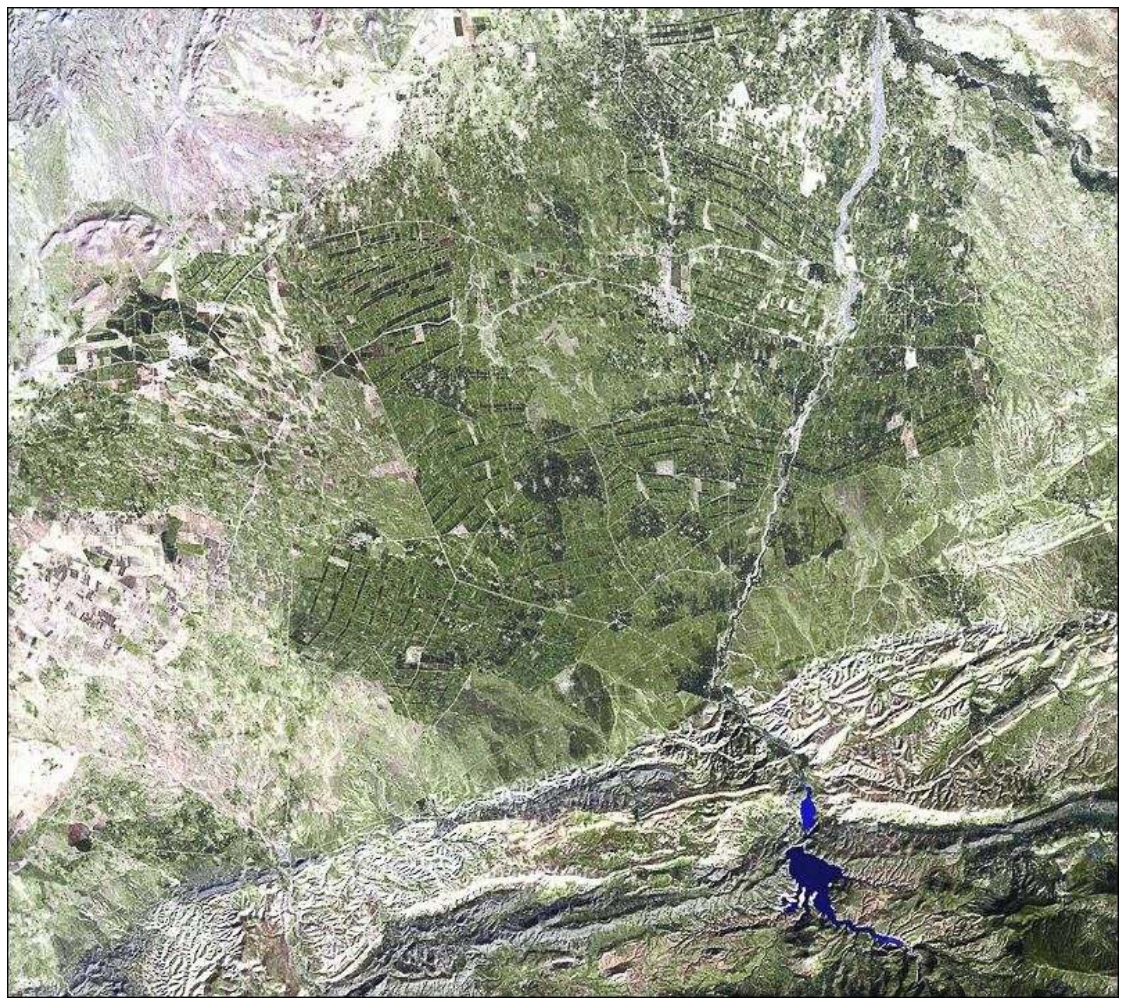

Dans ce mouvement global de modernisation «lourde", la région centrale de la Tessaout devient la Haute Tessaout. La coupure par le barrage provoque chez les ingénieurs hydrauliciens une sorte d'oubli de la vraie haute vallée située dans le Haut Atlas. La source du système est désormais le réservoir et l'usage de l'eau est concentré sur la plaine. Ainsi, le nouvel aménagement commande toutes les allocations d'eau depuis la première prise d'Agadir Bou Achiba jusqu'à la grande galerie d'adduction. À partir de là, la distribution de l'eau est calculée pour donner les mêmes volumes attribués à chaque bloc (voir les lignes rouges sur les illustrations 10 et 11). Nous allons découvrir que les nouvelles branches croisent partout les anciennes (les mesrefs). Ce nouveau découpage du territoire hydraulique local implique une perte de référence pour les usagers de l'ancien découpage : il n'y a plus de prises d'eau dans la rivière (elles ont même été détruites), et il n'y a plus le maillage hydraulique ancien sur lequel reposaient les tours d'eau. L'eau arrive désormais selon les volontés de l'administration et la distribution se fait en relation avec le plan de cultures qu'elle impose. Pendant quinze années, le nouveau système fonctionne "gratuitement", sans redevance ni taxe. L'administration hydraulique estime qu'en échange de la suppression totale des droits d'eau, une compensation est nécessaire durant une phase de transition. Cependant, une fois les quinze années passées, les usagers ont été tenus de payer une redevance à l'office, mais il semble que la collecte d'argent ne fut pas facile à faire. Dans les années 1990, sous l'impulsion de la Banque mondiale, la création d'associations d'usagers de l'eau agricole devait améliorer le fonctionnement général en laissant un peu d'autonomie pour assurer une meilleure collecte financière. Cette nouvelle organisation était présentée comme le meilleur concept pour moderniser les réseaux d'irrigation. Or, nous découvrons que $40 \%$ du territoire hydraulique de la Tessaout Amont ne fonctionne pas comme ce qui était présenté dans les documents de projet. La redistribution des terres selon le principe des trames B régulières n'a pas été radicale 
partout. Autour des anciens villages, les haouz de douar ont été épargnés et restent toujours aujourd'hui en dehors du jeu complexe de la planification de l'eau. À l'entrée de ces anciens terroirs où les vergers d'oliviers dominent dans un canevas foncier morcelé et diversifié, l'office d'irrigation délivre un montant global d'eau pour tout le secteur non remanié. Les agriculteurs utilisent encore leurs propres règles internes pour se partager cette eau. Ils font une collecte d'argent pour payer la redevance d'ensemble à l'administration.

Illustration 11 - Détail de la carte de l'illustration 10b

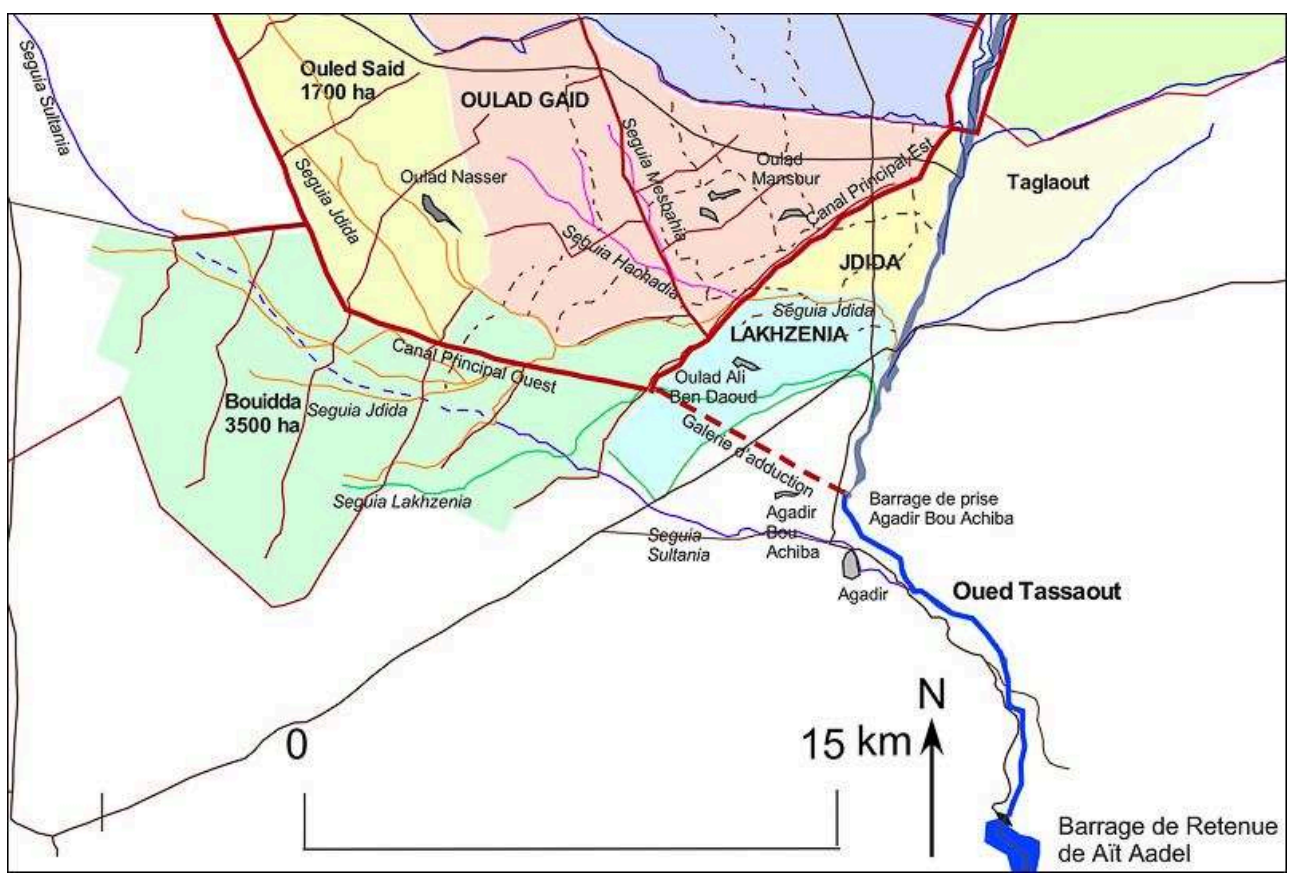

Comparaison entre les réseaux anciens de distribution (mesrefs colorés des seguias) et le nouveau réseau de distribution bétonné (nouvelles branches rectilignes rouges). Dans de nombreux terroirs villageois, le nouveau réseau n'a plus aucun lien avec l'ancien. 
Illustration 12 - La seguia Sultania, un cas d'étude de la Tessaout amont

Le canal du Sultan (seguia Sultania) est l'un des exemples d'aménagement hydraulique précolonial étudiés dans les archives de l'ORMVAH. Il existait déjà au XIXe siècle et il a traversé toutes les époques coloniales et celles des remaniements après l'Indépendance du Maroc. Il est l'œuvre du Sultan du Maroc Abd Ar Rahman qui régna de 1859 à 1873. En réalité, c'est plutôt l'achèvement d'un ouvrage déjà existant afin d'exploiter, au profit de l'État, de nouvelles terres du côté de Tamelelt. Il fit participer aux travaux plusieurs communautés de l'Atlas en échange de l'accès au quart des terres bonifiées et de droits d'eau.

En 1908, les terres de Tamelelt furent cédées au Pacha de Marrakech. Quelques années plus tard, il favorisa l'installation d'agriculteurs français. En 1924, ceux-ci disposaient déjà de la moitié des droits d'eau sur le canal. La politique coloniale était d'installer ici des soldats qui avaient participé à la première Guerre mondiale. L'administration du protectorat avait alors défini un mode de gestion de l'eau avec une ASAP, association syndicale d'agriculteurs privilégiés. Cependant, les choses n'allaient pas de soi. L'état du canal était déplorable. Les nouveaux agriculteurs français souffraient de sécheresses successives et du manque d'accord avec les usagers indigènes d'amont et même avec le Pacha. Les révoltes des populations locales incitèrent les autorités coloniales à améliorer le système hydraulique. Dans les années 1930, un groupe d'ingénieur du Génie rural entreprit le projet de modernisation du canal principal. Ils changèrent également l'organisation d'autres canaux utilisés par des groupes indigènes (seguia Jdida, seguia Lakhzeinia). En cas d'interruption du service de l'eau sur la seguia Sultania, les deux autres canaux devaient être utilisés à titre de secours (Kleiche-Dray, 2005).

Cette expérience d'intervention directe des ingénieurs hydrauliciens dans la gestion et les partages d'eau locaux fut généralisée un peu partout, lorsque les fermes coloniales étaient à proximité des aménagements hydrauliques anciens. Ce comportement général influença nettement la dernière génération d'ingénieurs coloniaux mais aussi, après l'indépendance, deux ou trois nouvelles générations marocaines. L'eau devint l'élément clé du pouvoir de l'administration centrale. Quant aux ASAP, elles furent supprimées au début des années 1960. Durant les 40 années de gestion hydraulique coloniale, il n'y eu aucun essai d'adaptation du concept d'associations d'usagers de l'eau pour les communautés agricoles marocaines. Après l'indépendance, le modèle choisi par les nouvelles autorités fut le contrôle étatique à travers une réforme agraire et des coopératives d'État, très distinct du modèle français des associations syndicales d'irrigation (les associations syndicales autorisées, ASA) qui existait en France. Dans les systèmes irrigués indigènes, l'administration coloniale se borna à reconnaître et actualiser les droits d'eau (en les sous-estimant parfois). Ces textes et données servent de références que les parties prenantes se renvoient aujourd'hui dans les conflits sur l'eau. De fait, la reconnaissance des jmaâ comme institutions de base dans la régulation des droits fut rejetée sous le protectorat et est restée obscure après l'indépendance, puisque lui a été superposée un découpage administratif directement relié au pouvoir central.

\section{La gestion de la rivière Nfis River à l'ouest de Marrakech : autres cadres, autres combinaisons}

41 Le processus de modernisation de la plaine du N'fis débute dans les années 1980 alors que celui de la Tessaout s'achève (Jolly, 2000, 2001 ; Finet 2002 ; Rocle 2005). La zone du N'fis était aussi un très bel exemple d'organisation hydraulique ancienne, où différents codes ou règlements permettaient une répartition des eaux de la rivière en fonction de droits différenciés sur les prises d'eau. Lorsque les colonisateurs français arrivèrent sur place, ils découvrirent un amphithéâtre de territoires hydrauliques (illustration 13, zones bleues), avec un haut niveau de conflictualité entre les groupes d'utilisateurs des 
prises et des canaux. Certains de ces groupes étaient natifs de la région, d'autres avaient migré par décision du Sultan du Maroc. Sur le plan foncier, il y avait une juxtaposition de régimes de partage des eaux différents, des terrains appartenant au Pacha représentant le sultan, à l'armée, le domaine propre au Sultan, et différentes terres collectives. Les troubles sur la répartition de l'eau étaient nombreux, reflétant les défis entre autorités et tribus. La partie nord de ce "delta " intérieur était alors considérée comme des terres sèches «bour» (terres cultivées en mode pluvial) qui pouvaient recevoir épisodiquement le surplus d'eau de crue (illustration 13, zone beige).

\section{Illustration 13 - Carte du réaménagement du N'fis en 1941}

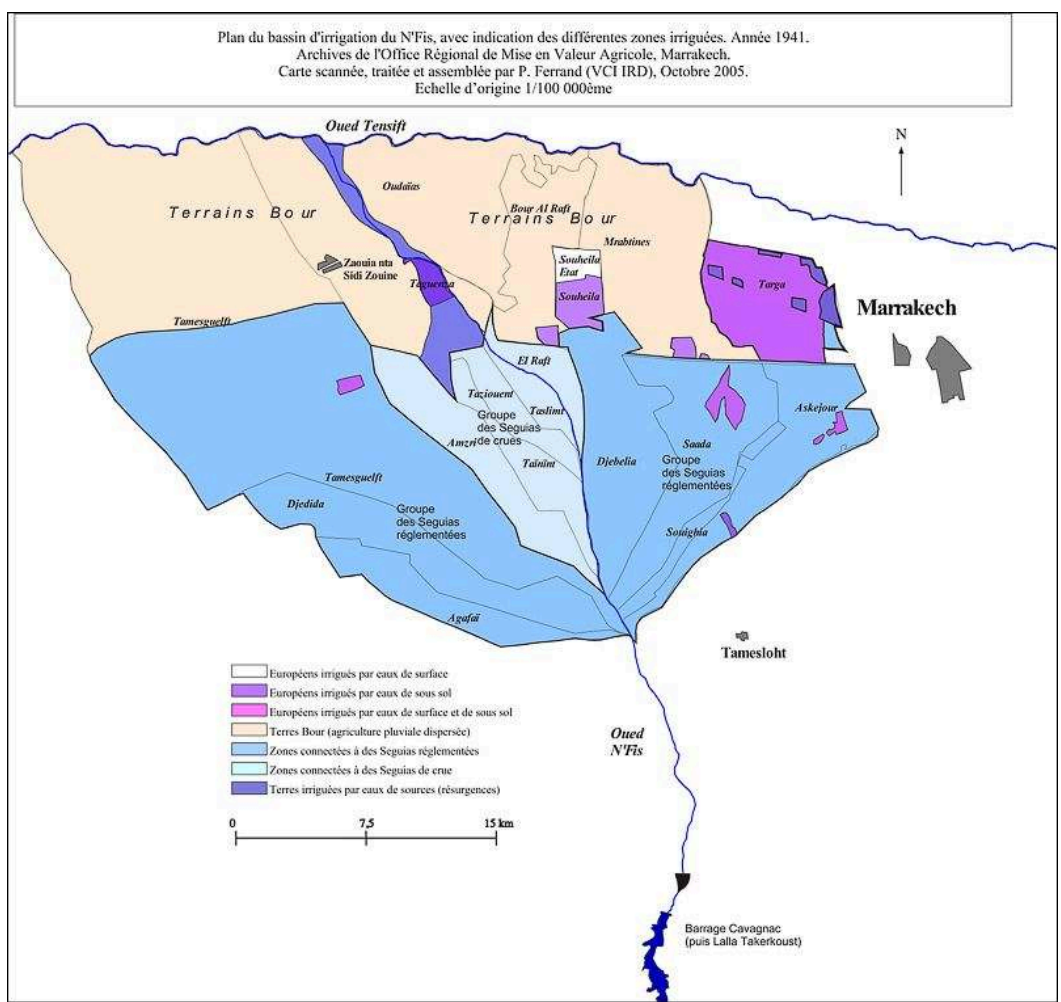

L'administration coloniale agit pour donner la réserve d'eau supplémentaire créée par le barrage aux seuls exploitants européens. Le transfert de l'eau se réalise par de nouveaux canaux de surface ou par la récupération des pertes d'eau dans les zones indigènes d'amont qui sont reprises par les colons situés plus bas, au nord de la plaine, et équipés de puits et de pompes puissantes.

L'administration hydraulique française organisa la collecte de nombreuses données sur les diverses situations locales et elle essaya de « rationaliser » les différentes pratiques des groupes d'usagers de l'eau. Ainsi, dans les années 1930, elle procéda à des mesures de débits des rivières et canaux, et des mesures de superficies des zones irriguées. Après une dizaine d'années de références, parfois moins, il fut décidé de classer les canaux en fonction de la position de la prise d'eau sur la rivière et du poids politique, démographique et économique des utilisateurs de l'eau. Les services hydrauliques coloniaux distinguèrent les canaux qui avaient la priorité de prélèvement pendant les étiages : on les désigna sous le nom de seguias d'hypothèque constante. En fonction de l'accroissement du débit dans la rivière, on pouvait ouvrir progressivement les prises des autres canaux ou au contraire, lorsque le débit diminuait les fermer selon un ordre précis. On désigna ces canaux de seguias de premier ou second ordre. Pour chaque canal, 
une nouvelle organisation des tours d'eau fut imposée pour fixer l'attribution de l'eau jour après jour à chaque village, branche par branche, mesref par mesref.

Illustration 14a - Le « delta intérieur » du N'fis

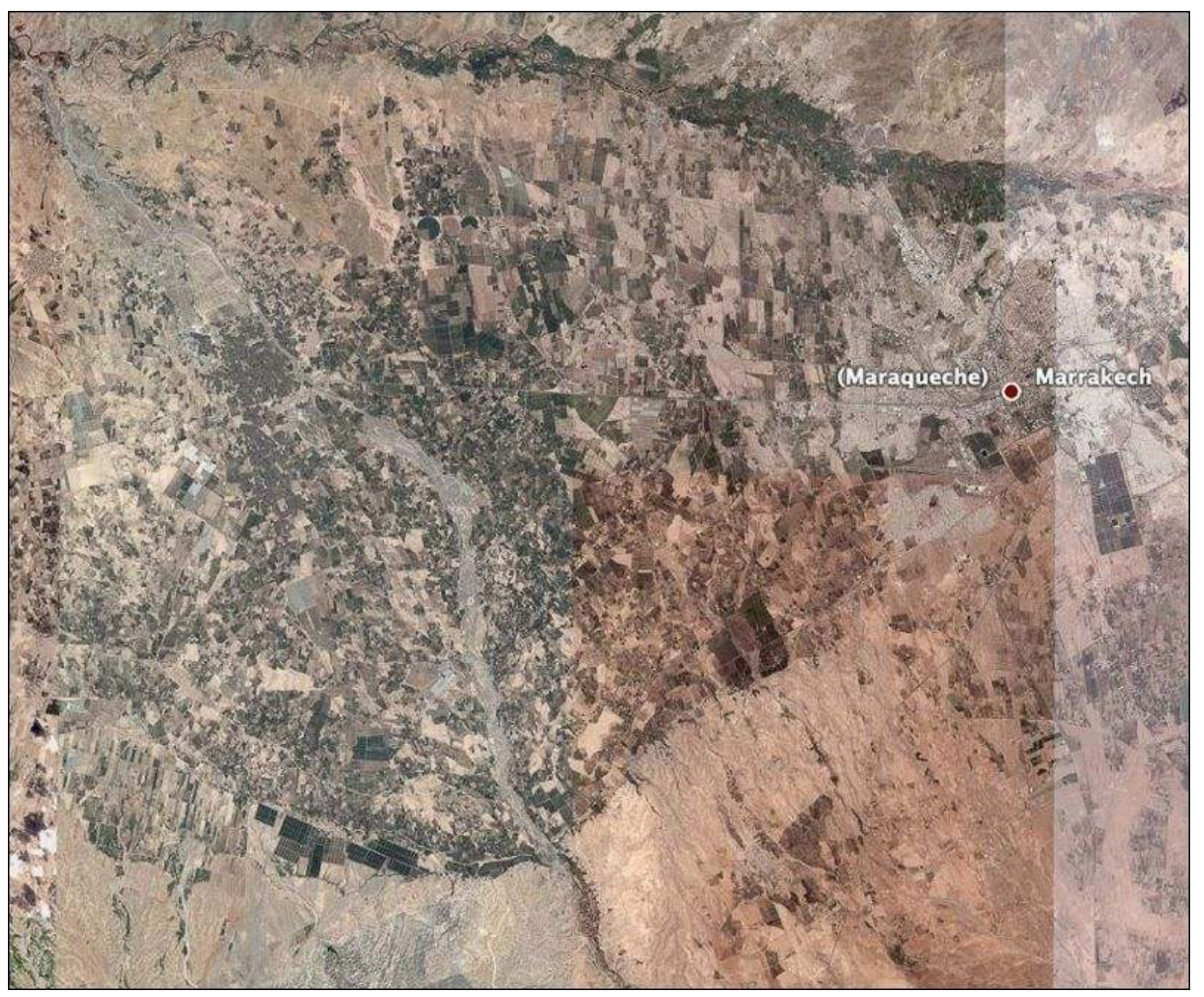

Source : Google Earth, 2009. 
Illustration 14b - Aménagement du Haouz Central, première tranche d'irrigation, schéma général du N'fis, juillet 1979

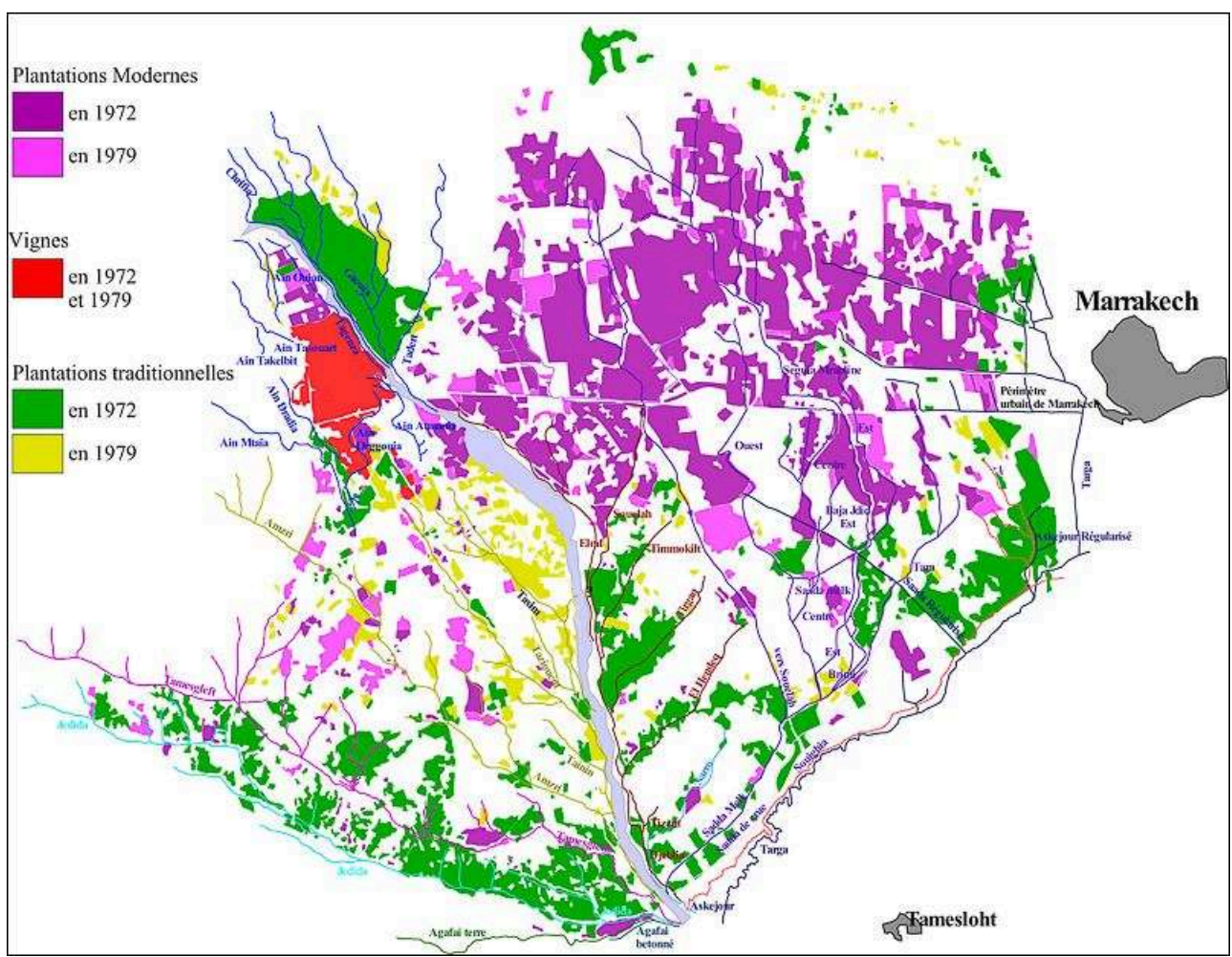

Les exploitations traditionnelles se trouvent au sud de la plaine, les grandes fermes issues des exploitations coloniales au nord (les zones claires correspondent au Bour ou cultures céréalières).

Sources: Compilation de plans n 1-5 au 1:50000 - Plantations existantes en 1972 et 1979 / Plan 1-4 Seguias existantes avant projet. Scans réalisés aux Archives de l'Ormvah le 4 octobre 2004 / Projet ISIIMM Maroc, M. Ait Bela - T.Ruf. 
Illustration 14c - Carte Aménagement du Haouz Central PTI. Étude du schéma général des secteurs

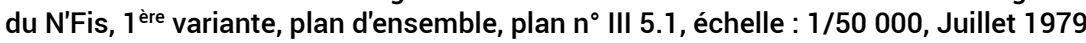

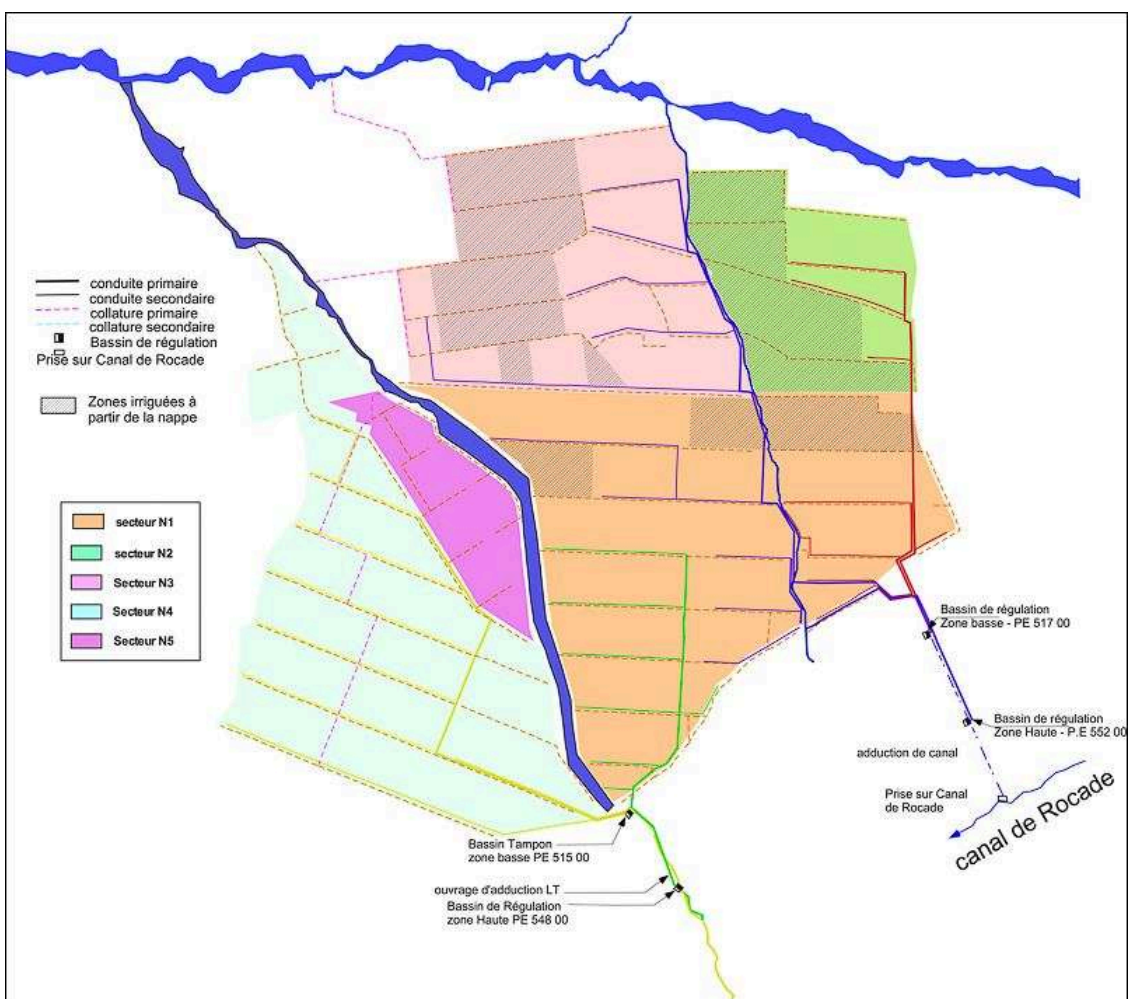

Projet envisagé de trame B selon l'expérience de la Tessaout.

Sources : Archives de l'Office régional de mise en valeur agricole du Haouz. Carte scannée par T. Ruf (IRD), assemblée et traitée par P. Ferrand (IRD), février 2005. 


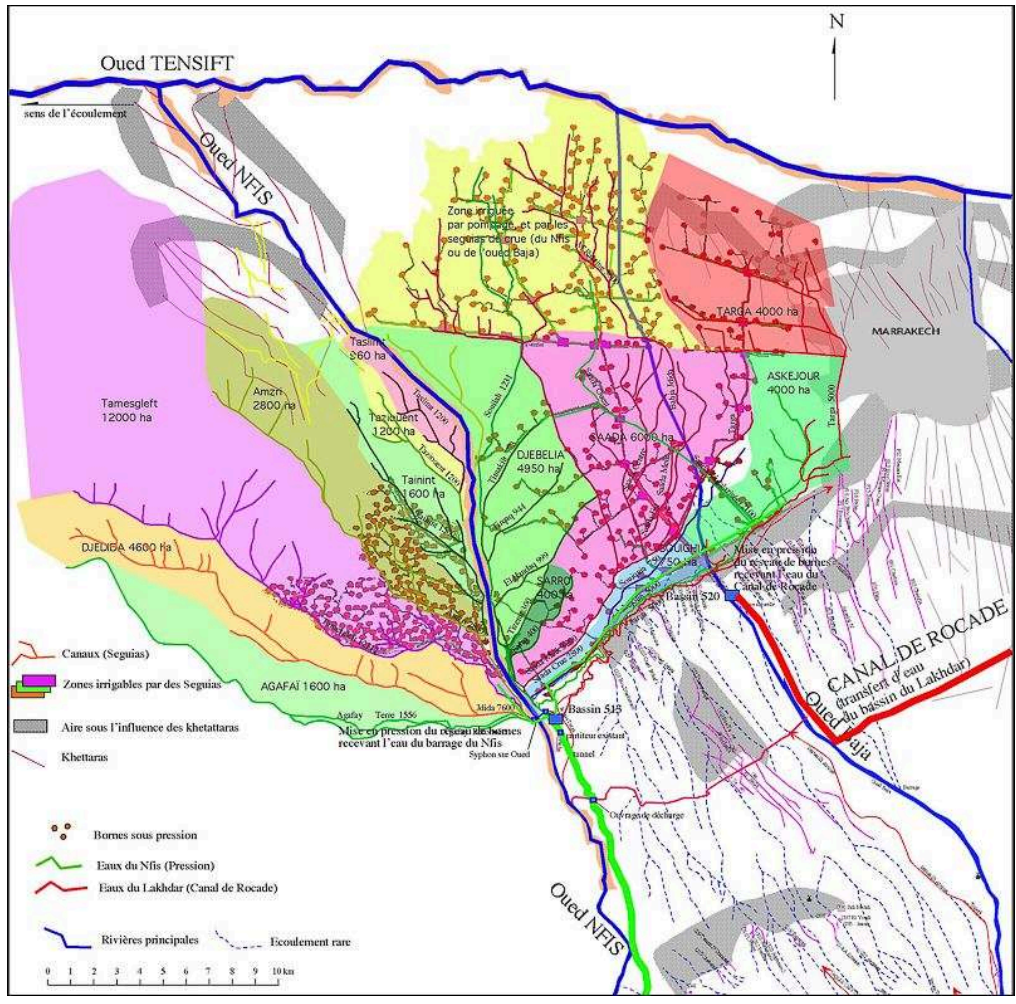

Les anciennes seguias sont figurées avec différentes couleurs. Le nouveau canal de Rocade est représenté en rouge, y compris les bornes. Les zones grisées étaient autrefois irriguées à partir de khettaras - dont la plupart ont été asséchés.

Source : El Faiz, Ruf, 2006

Pendant que tous ces éléments étaient remaniés, la politique d'installation d'agriculteurs européens se poursuivait dans la partie nord de la plaine, dans les espaces rarement irrigués par les seguias traditionnelles, sauf crue exceptionnelle. Les colons accédaient avant tout à l'eau de la nappe phréatique à partir de nombreux puits et de pompes. L'administration hydraulique décida d'établir un premier barrage réservoir sur le N'Fis et de mettre ainsi à leur disposition une eau plus abondante et moins coûteuse. En effet, la reconnaissance des droits des seguias indigènes était déterminée par le régime naturel de la rivière et notamment en période d'étiage. Le barrage permettait de créer un volume non attribué aux usagers anciennement installés, et qui pouvait du coup être largement attribué aux colons, à travers de nouveaux canaux bétonnés de contournement des zones irriguées indigènes. L'intérêt de ce transfert fut également reconnu dans certains rapports des ingénieurs: en réduisant les pompages dans la nappe souterraine, on avait plus de chance de conserver les niveaux piézométriques nécessaires au fonctionnement des khettaras. Cependant, à cette époque, les Européens eurent du mal à se débarrasser de leur individualisme qui contrastait avec les pratiques collectives des Marocains. Aussi l'exploitation de la nappe continua et le rabattement de la nappe commença à faire ses effets sur les galeries drainantes.

Après l'Indépendance, les exploitations coloniales furent attribuées à l'État et mises sous tutelle au Ministère de l'Agriculture ou bien vendues à de nouveaux propriétaires marocains (illustrations 14a et b). La demande en eau restait toujours un enjeu, si bien que le projet du transfert d'eau du Lakhdar vers le N'Fis via le canal de Rocade fut à 
nouveau promu par l'État marocain. À la fin des années 1970, l'achèvement des travaux du canal donna l'occasion aux ingénieurs de l'ORMVAH d'envisager de refondre totalement la trame foncière selon le modèle déjà fameux de la trame B. Cela concernait à la fois les secteurs irrigués «indigènes" comme ceux issus de la colonisation. Le rapport et la carte associée à ce projet de rationalisation totale ont été retrouvés aux archives de l'ORMVAH (Première variante, illustration 14c). Par la suite, il a été décidé de ne pas appliquer cette rationalisation agraire et de conserver la trame foncière ancienne, mais nous ne savons pas qui s'est opposé et qui a décidé de l'abandon du modèle de trame B pour l'office de Marrakech. Le schéma de modernisation repose alors sur un réseau à basse pression délivrant l'eau par des bornes dont les ouvertures et fermetures seraient déterminées en fonction de la demande (illustration 14d).

La mise en place de ce nouvel aménagement sur la rive droite de l'oued N'fis s'avère donc très différente de celle de la Tessaout. Les seguias anciennes et certaines khettaras sont encore actives après l'installation du réseau de l'office. Les prises d'eau en rivière des canaux restent fonctionnelles et jouent d'ailleurs un rôle très utile en temps de crue du N'fis, si le barrage est plein. En outre, les affluents peuvent parfois contribuer localement à la production de débit important. Pour éviter des destructions intempestives et profiter des effets positifs de ces apports, des institutions locales sont toujours prêtes à agir à temps, car situées à proximité des ouvrages et informées rapidement des évènements sur les lâchers et les écoulements occasionnels (Finet, 2002 ; Raki et Rocle, 2005 ; Yanogo, 2004).

46 En définitive, il est intéressant d'estimer, au sein de cette complexité hydraulique, quelles sont les contributions de chaque type de réseau. Il est en effet possible de dresser une carte combinant les informations sur les ressources en eau mobilisées et les formes d'institutions locales (administration publique pour les eaux du canal de Rocade et celles du barrage Lalla Takerkoust du N'fis, organisations coutumières jmaâ, pour la répartition des crues locales et des excédents du barrage, gestion privée de la nappe par des puits profonds ou des forages équipés de stations de pompage fonctionnant souvent avec du gaz en bouteilles subventionnées, pour des usages urbains en réalité). Pour que l'agriculture soit possible, il est préférable de combiner au moins deux types de ressources. Ainsi, les crises de l'eau autour de Marrakech sont principalement liées aux défauts conceptuels du cadre institutionnel, alors que l'on invoque souvent une origine climatique (El Faïz et Ruf, 2004).

Sur la rive gauche de l'oued N'fis, la situation est encore très différente. Dans les années 1990, l'administration hydraulique modifie le principe d'aménagement. On ne touche toujours pas à la trame foncière mais on opte pour un resserrement du réseau sous pression sur un espace limité, bien plus restreint que l'espace connecté aux seguias traditionnelles (illustration 15). En outre, dans cette zone modernisée, tous les blocs fonciers alimentés par une borne ont la même taille et donc reçoivent le même débit. En principe, cette zone ne doit plus recevoir aucune autre eau que celle apportée par le réseau de l'ORMVAH.

Pour le reste de l'aire connectée aux seguias de la rive gauche, l'office prévoie d'allouer de l'eau du barrage du N'fis selon les droits d'eau officiels (qui ont été définis à l'époque coloniale). En réalité, cet accès passe en second plan après le service offert au secteur modernisé situé à l'amont, dans la partie la plus haute de la plaine et la plus proche du barrage. 
49 Le canal de Tamesgleft est un bon exemple pur comprendre les conséquences du nouveau dispositif et ses règles. Conformément aux arbitrages donnés dans les années 1940, il reçoit l'eau du barrage en aval de deux autres canaux déjà servis. En principe, il s'agit d'un débit de 1000 litres par seconde pendant douze jours, chaque mois. Pour différentes raisons, ces lâchers ne sont réalisés que trois ou quatre fois par an, selon le plan de gestion du barrage qui dépend de la Direction nationale de l'hydraulique, dont le siège est à Rabat.

Le canal de Tamesgleft bénéficie dans les années 2000 du plan national d'irrigation qui stipule qu'il faut rapidement achever le programme du million d'hectares irrigués. L'ORMVAH inclut dans le projet de modernisation le revêtement en béton du canal principal et applique le changement de règles d'allocation des lâchers de douze jours. En 2004, l'Office interdit à la zone haute équipée de bornes sous pression d'accéder à l'eau du canal Tamesgleft. Le canal remodelé en béton ne permet plus de connecter les anciennes branches (mesref) des villages concernés. Il s'en suit en 2004 une violente confrontation avec les ayant-droits d'amont, qui cassent les parois du canal et rétablissent d'une manière assez rustique les anciens accès. Les usagers d'aval, ceux de la zone centrale desservie par le canal (zone jaune de la carte, illustration 15) préfèrent discuter avec ceux de l'amont, sur les changements d'allocation possibles, en incluant des transactions financières par achat de temps d'utilisation du débit du canal. Ils préfèrent des arrangements négociés entre eux plutôt que de perdre leur pouvoir avec une règle de tour d'eau imposée par une intervention de l'ORMVAH. Par ailleurs, une des raisons majeures du conflit est liée à la gestion des débordements du barrage Lalla Takerkoust et des crues des petits oueds qui alimentent en plusieurs points le canal de Tamesgleft. Lorsque le barrage déborde, les conventions entre les usagers de Tamesgleft stipulent que tous les mesrefs sont desservis de l'amont vers l'aval en partant bien du premier village. Cet accès avait été rendu impossible par le bétonnage complet des berges du canal et l'ouverture des brèches est finalement le rétablissement de ce droit. Lorsque les petits oueds entrent en crue, la gestion globale du canal s'arrête et chaque tronçon fonctionne indépendamment des autres. La solution au conflit passe finalement par un diagnostic partagé entre toutes les parties prenantes et le rétablissement des ouvrages de partage conformes aux règles et institutions locales. 
Illustration 15 - Carte des anciens et nouveaux aménagements de la rive gauche de l'oued N'fis en 2006

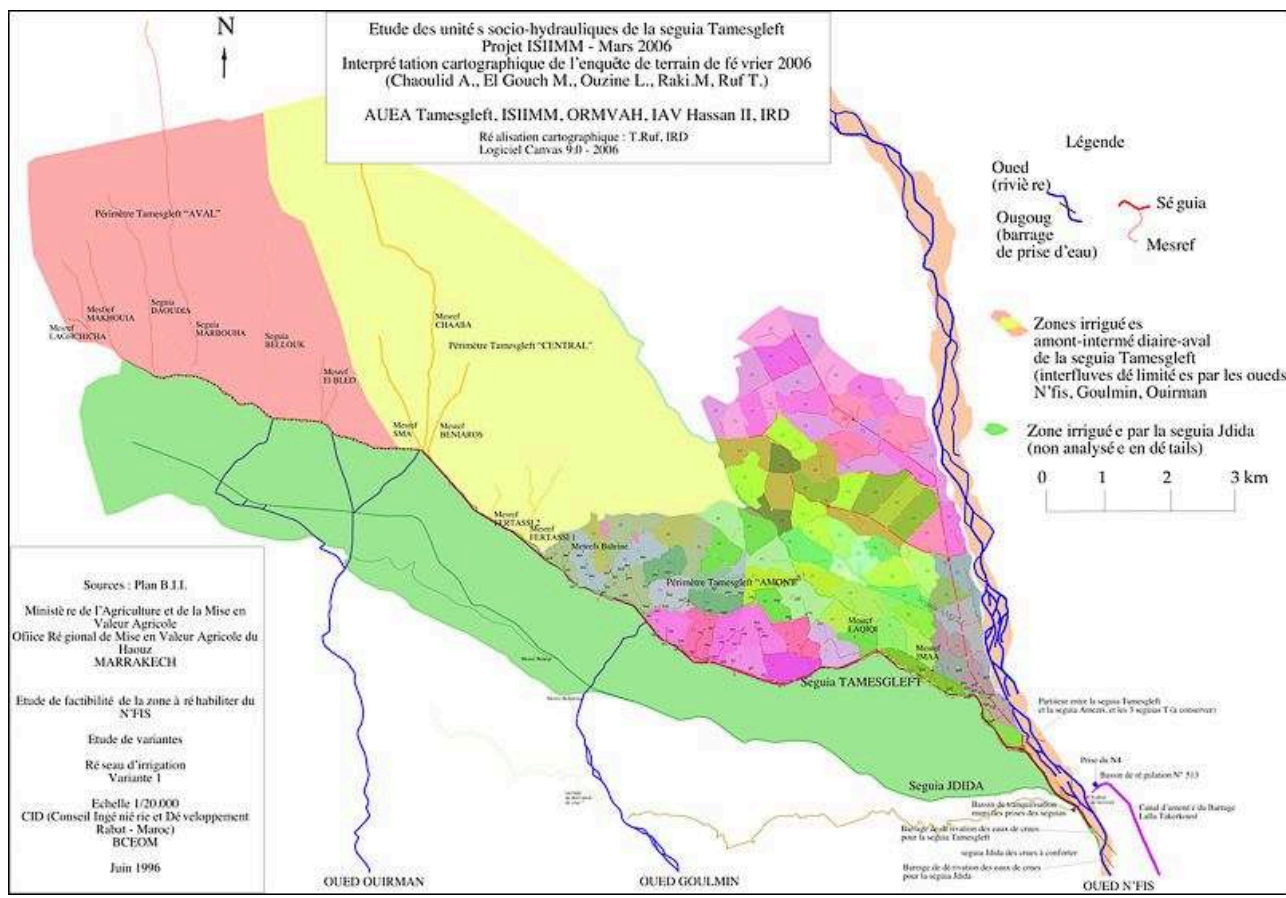

Superposition de systèmes et rivalités sur les différentes ressources : eau venant de l'oued N'fis, eau venant des petits affluents, eau venant du barrage et à côté des systèmes de surface, eau souterraine extraite par des stations de pompage privées.

\section{Conclusion}

Dans les années 1980, Paul Pascon avait montré que la société marrakchi était configurée par la question hydraulique, et que cette structure économique, sociale et politique atténuerait la domination d'un modèle de développement agricole capitaliste. Quarante ans après, le Haouz de Marrakech reste une composition contrastée où coexistent toujours différents modèles économiques et sociaux. Certes, les intérêts privés à proximité de la ville ont pris le dessus, comme par exemple l'installation spéculative de 18 golfs, ou la multiplication des exploitations agricoles. Certes les khettaras ont quasiment totalement disparu, autant par l'impossible défense collective contre les milliers de forages et de pompages que pendant longtemps personne n'a souhaité limiter, que par la difficulté à coordonner les centaines de communautés d'usagers de l'eau des khettaras affectées. Cependant, en dehors de l'espace urbanisé ou en voie d'urbanisation, la quête de l'eau reste toujours un mixte entre les eaux de seguias gérées en bien commun, les eaux de barrage administrées par l'office du Haouz et les eaux souterraines exploitées en fonction des moyens disponibles des particuliers. Dans la Tessaout, le Haouz central ou le N'fis, la planification centrale rigide côtoie les transactions locales de toutes sortes sur les eaux superficielles comme sur les eaux souterraines. La mise en place des associations d'usagers de l'eau agricoles est restée formelle en Grande Hydraulique où pratiquement aucune responsabilité n'a été transférée, tandis qu'elle serait plus tangible dans les zones de PMH.

De nos jours, l'ORMVAH travaille dans des conditions difficiles pour maintenir son leadership sur les ressources en eau. Les aménagements vieillissent et subissent de 
nombreux dysfonctionnements. La planification des cultures est un souvenir, les producteurs choisissent leurs productions comme ils l'entendent et comme ils le peuvent quand les contraintes sont fortes. Les plus importants gèrent librement le quota de l'eau des barrages de l'État mais en fait, utilisent surtout leurs très grandes capacités de pompage et surexploitent l'eau souterraine (Tanouti, 2017). Les petits producteurs sont marginalisés après 40 années de pression bureaucratique. Ceux qui poursuivent leurs activités le font souvent en gérant l'eau de petits oueds non équipés de barrage et pour lesquels l'office n'est pas impliqué jusqu'à présent.

Malgré ce panorama sombre de la situation, on peut penser que des solutions existent pour tenter de concilier les antagonismes et refonder des territoires hydrauliques viables en mutualisant les différentes ressources et en instituant une gestion juste, équitable et opérante de l'eau avec de véritables associations assises sur un périmètre défini, avec des règles constitutionnelles et opérationnelles claires. Du concept de Grande Hydraulique, on pourrait envisager celui de la gestion sociale de l'eau (Ruf, 2017) dans un maillage redessiné, un compromis entre intérêts publics, privés et collectifs. En tous cas, début 2018, les principaux acteurs publics ont signé une charte pour reprendre la question hydraulique et éviter l'épuisement de la nappe phréatique. Ils l'ont fait dans le cadre d'une institution nouvelle apparue en 2017 sur la scène marrakchi : le musée Mohamed VI pour la civilisation de l'eau au Maroc présente de manière très pédagogique, sur un espace conséquent de près de 3000 mètres carrés les différentes manières d'aborder l'eau sous l'angle de l'écologie et des sciences humaines, autour des régions porteuses de civilisation de l'eau, les oasis, les montagnes, le littoral et le Haouz de Marrakech. Le musée appartient au ministère des Habous, qui exerçait avant 1912 un rôle de gestionnaire de l'eau dans les villes et d'arbitre dans la répartition des eaux. Devenir un centre d'interprétation des questions de l'eau au Maroc est un objectif affiché par le Ministre des Habous, le Pr Ahmed Toufik.

\section{BIBLIOGRAPHY}

Ait Kadi M., 1995. La maîtrise des demandes en eau dans les grands périmètres irrigués. Spécificités des grands aménagements hydro-agricoles au Maroc. Séminaire national sur la gestion participative de l'irrigation dans le Royaume du Maroc. 10 p.

Aderghal M., Simenel R., 2012. La construction de l'autochtonie au Maroc. Espace populations sociétés [En ligne], 2012/1 ; http://journals.openedition.org/eps/4847 ; DOI : 10.4000/eps.4847

Attar H., Ben Mohamed N., 1987. Spécial : l'hydraulique agricole contemporaine au Maroc. Hommes, Terre et eaux - Revue marocaine des sciences agronomiques et vétérinaires, vol. 17, n 65/66.

Berriane Y., 2015. Inclure les « n'ayants pas droit »: Terres collectives et inégalités de genre au Maroc ». L'Année du Maghreb, $\mathrm{n}^{\circ}$ 13. http://journals.openedition.org/anneemaghreb/2546 ; DOI : 10.4000/ anneemaghreb.2546

Bolens L., 1981. Agronomes andalous du Moyen-Age. Genève, Droz, 305 p. 
Bouderbala N., Chiche J., Herzenni A., Pascon P., 1984. La question hydraulique, 1. Petite et moyenne hydraulique au Maroc. IAV Hassan II, Rabat, 397 p.

Bouderbala N., 1987. Logique foncière de l'État et logiques foncières des exploitants dans les grands périmètres d'irrigation au Maroc. Aménagements hydro-agricoles et systèmes de production. Actes du troisième séminaire 16-19 décembre 1986, collection Documents Systèmes Agraires, $\mathrm{n}^{\circ}$ 6, Montpellier, CIRAD, p. 343-350.

Bouderbala N., 1996. Les terres collectives du Maroc dans la première période du protectorat (1912-1930). Revue du monde musulman et de la Méditerranée, n 79-80, p. 143-156.

Bouderbala N., 1999. Les systèmes de propriété foncière au Maghreb. Le cas du Maroc. Politiques foncières et aménagements des structures agricoles dans les pays méditerranéens. À la mémoire de Pierre Coulomb, Cahiers Options Méditerranéennes, vol. 36, Paris, CIHEAM, p. 47-66.

Bouderbala N., Chraïbi M., Pascon P., 1974, 1977. La Question agraire au Maroc. Bulletin économique et social du Maroc, $\mathrm{n}^{\circ} 123-124-125,1974$, et $\mathrm{n}^{\circ}$ 133-134, 1977.

Debbarh A., Badraoui M., 2002. Irrigation et environnement au Maroc : situation actuelle et perspectives. Vers une maîtrise des impacts environnementaux de l'irrigation, Actes de l'atelier du PCSI, 28-29 mai 2002, Montpellier, CEMAGREF, CIRAD, IRD, Cédérom du CIRAD.

Ducrocq M., Zerhouni M., 1987. Aménagement du périmètre du N'fis, Haouz de Marrakech, un essai d'adaptation d'aménagement aux conditions locales. Aménagements hydro-agricoles et systèmes de production, Actes du troisième séminaire 16-19 décembre 1986, Collection Documents Systèmes Agraires n 6, Montpellier, CIRAD, p. 331-341.

El Faïz M., 1999. Les aménagements hydro-agricoles dans le grand Haouz de Marrakech : histoire d'une idée. Cultures, usages et stratégies de l'eau en Méditerranées Occidentales. Tensions, conflits et régulation, Paris, L'Harmattan, p. 59-78.

El Faïz M., 2001. La Grande Hydraulique dans le Haouz de Marrakech : fascination technologique et émergence du pouvoir des ingénieurs In Gobe É. (dir.). Les ingénieurs maghrébins dans les systèmes de formation. Tunis, Institut de recherche sur le Maghreb contemporain, 2001.

El Faïz M., 2005. Les maîtres de l'eau. Histoire de l'hydraulique arabe. Actes Sud, Arles, 363 p.

El Faïz M., Ruf T., 2006. La gestion collective de l'eau est-elle encore possible dans le Nfis à l'Ouest de Marrakech? Coordinations hydrauliques et justices sociales, $4^{\mathrm{e}}$ séminaire du PCSI Agropolis Montpellier, 25 et 26 novembre 2004, CIRAD, www.hal.archives-ouvertes.fr/docs/ 00/15/43/99/.../El_FaizRuf_VF2.pdf

El Haouari N., 2003. Case study from Morocco - Survey on irrigation modernization: Tessaout Amont Haouz, http://www.fao.org/landandwater/aglw/watermanagement/docs/MOD_Morocco.pdf

En-Naciri, A. K., 1906-1907. Kitab Al-Istiqça li ahbar duwal Al Maghreb Al Aqsa. Archives Marocaines, Paris, Ernest Leroux.

Finet A., 2002. Diagnostic des systèmes de production du périmètre irrigué du N'fis (Maroc) : un aménagement aux résultats contradictoires. Mémoire ESAT 1, Montpellier, CNEARC, 81 p.

Ftaïta T., 2012. De la gestion communautaire à la gestion associative de l'eau. Légitimité et illégitimité des associations des usagers des eaux agricoles comme nouveau cadre institutionnel de la gestion participative au Maroc. In Aspe C., De l'eau agricole à l'eau environnementale. Quae, p. 351-362.

Hardin G., 1968. The tragedy of the commons. Science, $\mathrm{n}^{\circ}$ 162, p. 1243-1248. 
Herzenni A., 1975. Problèmes d'aménagement des structures foncières dans le périmètre d'irrigation de la Tessaout Amont. Hommes Terres et Eaux - Revue Marocaine des Sciences Agronomiques et Vétérinaires, vol. 4, n 15, juin 1975.

ISIIMM, 2003-2008. Innovations Sociales et Institutionnelles dans la Gestion de l'eau en Méditerranée. http://www.isiimm.agropolis.org - documents en ligne.

Jolly G. (dir.), 2000. Gestion sociale de l'eau dans le N'fis et mise en place des associations d'usagers de l'eau agricole. Rapport de synthèse, Montpellier, CNEARC, 64 p.

Jolly G., 2001. La gestion des périmètres irrigués, méthodologie de diagnostic : cas d'un transfert de gestion : les associations d'usagers du périmètre du N'fis (Office du Haouz, Maroc). La gestion des périmètres irrigués collectifs à l'aube du XXI siècle : enjeux, problèmes, démarches, Actes de l'atelier du PCS, p. 25-46.

Kleiche-Dray, M., 2001. Aux origines du concept de développement. Quand l'irrigation devient enjeu de réforme agricole : nouvelle mise en ordre du paysage rural marocain dans les années 1930. Hespéris Tamuda, 39 (2), p. 175-195.

Kleiche-Dray M., 2005. Les ingénieurs et la modernisation de l'irrigation au Maroc : analyse de la transformation de la seguia Sultania dans les années 1930. $4^{e}$ Conférence IWHA UNESCO, L'eau et civilisation, Paris, 1-4 décembre.

Kleiche-Dray M., 2007. The Office Régional de Mise en Valeur du Haouz Archives: Testimonies of modern irrigation introduction in the twentieth century Morocco. IWHA 2007 Pasts and Futures of Water, Tampere, 13-17 june.

Lahlimi A., 1964. Droits d'eau dans la Tessaout - secteur hydraulique amont. Archives de l'ORMVAH Marrakech.

Lebahers G., 2006. Gestion sociale de l'eau comparée entre réseaux anciens et modernes dans la Tessaout Amont (Maroc). CNEARC, GSE, FSJES Marrakech, IRD, ORMVAH, 154 p.

Louhmadi B. 1996-1997. Archéologie hydraulique : canalisations et anciennes structures agraires dans le bassin de la Tassaout aux époques médievale et moderne (Maroc Cnetral). Paris, Université de Paris I Panthéon- Sorbonne.

Mahdi M., 2009. La tribu au secours du développement pastoral. Études rurales [En ligne], 184 .URL : http:// etudesrurales.revues.org/10531

Mellakh K, 2004. La borne d'irrigation serait-elle un lieu d'exclusion sociale dans le Haouz de Marrakech ? Coordinations hydrauliques et justices sociales, $4^{e}$ séminaire du PCSI Agropolis, Montpellier, 25 et 26 novembre 2004, CIRAD, www.hal.archives-ouvertes.fr/docs/00/15/43/15/PDF/

Mellakh.pdf

ONI (Office National des Irrigations), 1964. Projet d'aménagement et de mise en valeur du secteur hydraulique de la Tessaout Amont - Étude préliminaire - Rapport de synthèse. Archives de l'ORMVAH Marrakech.

ORMVAH, 1967-1968. DOH Remembrement rural Tessaout Amont 1967 - 1968. Archives de l'ORMVAH Marrakech.

Ostrom, E. 1992. Crafting Institutions for Self-Governing Irrigation Systems. San Francisco, ICS Press, $111 \mathrm{p}$.

Pascon P., 1968. Note de réflexion au sujet du projet de création d'ASAP dans la R.D. de la Tessaout. Marrakech, ORMVAH, 4 p, 26 octobre 1968.

Pascon P., 1977. Le Haouz de Marrakech. Rabat, CURS, CNRS, INAV, 2 tomes, 694 p. 
Pascon P., 1984. La petite et moyenne hydraulique au Maroc. Problèmes institutionnels et juridiques posés par son extension au Maroc. La question hydraulique, tome 1. S.N. p. 443-476.

Pop H., 1984. Effets socio-géographiques de la politique des barrages au Maroc. La question hydraulique, tome 2, IAV Hassan II, Rabat, 266 p, + cartes.

Rachik H., Bourqia R. 2011. La sociologie au Maroc. SociologieS [En ligne], URL : http:// journals.openedition.org/sociologies/3719

Raki M., Ruf T., 2006. La participation des usagers de l'eau des périmètres irrigués méditerranéens : regards croisés sur deux modèles marocain et égyptien. Coordinations hydrauliques et justices sociales, $44^{e}$ séminaire du PCSI Agropolis, Montpellier. 25 et 26 novembre 2004, CIRAD. www.hal.archives-ouvertes.fr/docs/00/15/43/70/PDF/Raki-Ruf-VF.pdf

Rocle N., 2005. Gestion des eaux et superposition de réseaux sur la seguia Jbelia, périmètre du Nfis, Haouz de Marrakech. ENGEES- CNEARC-GSE, FSJES Marrakech, IRD, ORMVAH, 165 p.

Roussillon, A. 2002. Sociologie et identité en Égypte et au Maroc : le travail de deuil de la colonisation. Revue d'Histoire des Sciences Humaines, 2002/2, $\mathrm{n}^{\circ} 7$,

p. 193-221.

Ruf T. (coord.), 2009. Histoire comparée des irrigations en Méditerranée. Éclairage des archives pour un développement durable. Rencontre internationale finale du programme« Archives de l'irrigation en Méditerranée » (AIME) Programme mobilisateur d'appui à la coopération pour la recherche en sciences humaines et sociales entre le Maghreb et la France (Ministère français des Affaires Etrangères et Européennes, Maison des sciences de l'Homme), 8 - 9 octobre 2009, Rabat, IRD, Université de Marrakech, Bibliothèque Nationale du Royaume du Maroc, 48 p.

Ruf T., 2011. Le façonnage des institutions d'irrigation au XX ${ }^{\mathrm{e}}$ siècle, selon les principes d'Elinor Ostrom, est-il encore pertinent en 2010 ? Natures Sciences Sociétés, $n^{\circ}$ 19, p. 395-404.

Ruf T., 2017. L'accès à l'eau, une question de justice pluriscalaire. In Chataigner J-M., Les objectifs du développement durable, Marseille, IRD, chap. 17, p. 161-169.

Simonneaux V., Lepage M., Helson D., Metral J., Thomas S., Duchemin B., Cherkaoui M., Kharrou H., Berjami B., Chehbouni A., 2009. Estimation spatialisée de l'évapotranspiration des cultures irriguées par télédetection : application à la gestion de l'irrigation dans la plaine du Haouz (Marrakech, Maroc). Sécheresse, vol. 20, n 1, janvier-février-mars 2009, p. 123-130.

Swearigen W. D., 1987. Moroccan Mirages: Agrarian Dream and Deceptions 1912-1980. Princeton, Princeton University Press, 218 p.

Tanouti O., 2017. La gestion intégrée des ressources en eau à l'épreuve du bassin versant - cas du bassin du Tensift au Maroc. Université de Paris Nanterre, 399 p.

Tozy M., 2013. Paul Pascon : un pionnier de la sociologie marocaine. SociologieS [En ligne], URL : http://journals.openedition.org/sociologies/4322

Wittfogel K., 1957. Oriental despotism, a comparative study of total power. Yale Univ. press. Ed. française utilisée: Le despotisme oriental, Editions de Minuit, 1977, 651 p.

Yanogo A.A., 2004. Gestion participative de l'irrigation dans le périmètre du N'Fis (ORMVA du Haouz) : cas des AUEA Tizemt et Tazakourt. Quel partenariat ORMVAH-Usagers dans la gestion de l'irrigation ? Rabat, IAV Hassan II, Département des sciences humaines, III ${ }^{\mathrm{e}}$ cycle ingénieur d'État en Agronomie, $213 \mathrm{p}$. 


\section{NOTES}

1. «Haouz » signifie l'espace périphérique de la ville, c'est à dire une banlieue campagnarde agropastorale (Pascon, 1983).

2. Plusieurs articles sont revenus ces dernières années sur la place des travaux de Pascon dans l'émergence d'une sociologie critique et engagée au Maroc, voir Roussillon, 2002 ; Rachik \& Bourquia, 2011 ; Tozy, 2013.

3. Paul Pascon donne une définition contextualisée de ces termes à partir des travaux de terrain qu'il mène en particulier dans le Haouz, mais sur lesquels nous ne reviendrons pas ici, voir sa thèse, (Pascon, 1977).

4. Il s'agit des us et coutumes.

5. Litt. : magasin, entrepôt. Plus que l'État lui-même ou l'administration, ce terme désigne le système d'emprise du pouvoir central sur la société, voir Laroui, 1977 cité par Roussillon, 2002 p. 198.

6. Au Maroc, le développement de l'irrigation sous forme d'aménagements de grande ampleur remonte certainement à la dynastie des Almoravides (1053-1124) et à celle des Almohades (1124-1269) (En-Naciri 1906, 1907 ; Bolens, 1981 ; El Faiz, 1993 ; Louhmadi, 1996-1997). Cependant, des travaux hydrauliques ont été réalisés bien auparavant, notamment à Volubilis, mais ils semblent avoir eu une orientation urbaine et un développement limité dans l'espace. Les canaux établis du XIe au XIIIe siècle s'inspiraient de ceux qui avaient été entrepris en Andalousie autour des principales villes arabo-andalouses. Au XVI siècle, une nouvelle période d'expansion de l'irrigation survient avec l'arrivée des Maures fuyant l'Espagne.

7. La définition de groupes ethniques et/ou tribus qui a été utilisée depuis la période coloniale a été à maintes fois remise en cause et est actuellement toujours en débat autour de la question de la revitalisation des langues, des revendications territoriales notamment à partir de la redistribution des terres collectives. C'est pourquoi nous parlerons ici plutôt de groupes de populations qui se sont inscrits historiquement dans des territoires aux contours flexibles reposant sur la gestion d'un espace ouvert dans lequel un groupe dispose de l'ensemble des ressources nécessaires à son existence (forêts, eau, terres cultivables, parcours) et passe des accord avec les groupes voisins pour l'accès à des ressources complémentaires, voir Bouderbala, 1996 ; Aderghal \& Simenel, 2012 ; Berriane, 2015.

8. Biens dont la succession est immobilisée dans un but de piété et affectée à une utilisation collective.

9. Sur les différents statuts des terres au Maroc et leurs évolutions historiques, voir les remarquables travaux de Bouderbala, notamment Bouderbala, 1996, 1999.

10. Durant le protectorat, les collectivités foncières organisées autour des jamaâ avaient été mises sous tutelle de la Direction des Affaires Indigènes à partir de 1919 puis de la Direction des Affaires Politiques à partir de 1937. Nous utilisons alors ici le terme « indigène » comme catégorie construite durant la période coloniale pour désigner les personne appartenant à des collectivités durant la période coloniale qui ont fait l'objet de mesures d'exception le plus souvent discriminatoires au regard du droit français. Sur la question de l'indigénat dans l'Empire colonial français, voir Merle, 2004.

11. Statut désignant historiquement les terres octroyées à l'armée par le Sultan.

12. Melk : propriété privée familiale de droit musulman voir les travaux de Bouderbala cités en bibliographie.

13. Quelques années après, on appellera cette zone la «Tessaout amont », curieuse dénomination puisque la véritable région amont de la Tessaout se trouve en fait dans le Haut Atlas, en amont du barrage réservoir. 
14. Ce terme désigne une politique de portée générale mise en place par le gouvernement marocain en 1973 pour récupérer les dernières terres, les entreprises qui avaient été maintenues entre les mains de nombreux ex-colons après l'indépendance.

\section{ABSTRACTS}

Dans le monde de l'irrigation, la région de Marrakech est connue pour l'ancienneté et la complexité de réseaux de canaux de surface, les seguias, alimentés par les oueds du Haut Atlas et de réseaux de galeries drainantes, les khettaras, qui ramènent en surface les eaux de la nappe phréatique. Dans les années 1970, la gestion de ces ouvrages hydrauliques suivait différents modèles: la gestion coutumière, el 'urf, qui a subi des modifications à la fois du temps du protectorat et après l'indépendance, la gestion publique initiée par le protectorat puis élaborée à partir de barrages et de transferts d'eau réalisés par l'État marocain indépendant et la gestion privée liée aux décisions d'exploitation prises par des investisseurs pour pomper directement dans la nappe. Quarante ans après les travaux du sociologue Paul Pascon sur la société composite du Haouz de Marrakech, nous avons cherché ici dans la même veine à comprendre les évolutions spatiales et historiques de ces modèles de gestion, en particulier dans l'aire d'action de l'office régional de mise en valeur agricole du Haouz, dont P. Pascon fut l'un des premiers directeurs. L'étude a porté sur trois hydro-systèmes, celui de la Tessaout, du Haouz Central et du N'Fis dont les trajectoires diffèrent. Elle conjugue l'approche géographique précise des réseaux et l'analyse historique grâce aux archives anciennes que l'ORMVAH conserve à Marrakech. En instituant un service hydraulique d'État, le protectorat a figé les droits d'eau anciens pour en définir de nouveaux au profit des colons. Nonobstant, si les khettaras ont disparu par tarissement général du fait de la la surexploitation de la nappe, de nombreuses seguias communautaires continuent de fonctionner en 2018, sauf dans la Tessaout où l'administration marocaine a joué sur la rupture totale avec l'ancienne trame hydraulique et foncière. Le Haouz central dispose d'un outil hydraulique nouveau, le canal de Rocade, dont la gestion par quotas ne permet pas de répondre aux besoins des cultures irriguées. Cela a incité ceux qui en avaient les moyens à multiplier les forages dans une concurrence effrénée. Le N'Fis est l'archétype du maintien de la gestion plurielle du Haouz, où différents réseaux anciens et modernes se superposent pour gérer des eaux régulées publiques et communautaires, des eaux non régulées communautaires et des systèmes d'extraction privés dans la nappe. Nous montrons ici que tous ces réseaux continuent à être interdépendants malgré une absence de conciliation entre les différents acteurs. Cependant, leurs fonctionnements efficaces, justes et durables n'ont pu émerger ni dans la démarche bureaucratique historique, ni dans la course d'accaparement privée en cours aujourd'hui. Construire des communautés d'usagers de l'eau agricole sur la trame géographique des réseaux et prenant en compte leurs singularités historiques est un chantier encore à mener.

\section{INDEX}

Mots-clés: irrigation, Haouz, Marrakech, histoire, État, Communs

Subjects: Sur le Champ - Sur le Terrain 


\section{AUTHORS}

\section{THIERRY RUF}

Thierry Ruf, thierry.ruf@ird.fr, est géographe à l'IRD UMR GRED. Il a publié récemment :

- Ruf T., 2017. L'accès à l'eau, une question de justice pluriscalaire. In Chataigner J.-M., Les objectifs du développement durable, IRD, Marseille, Chap. 17 p. 161-169.

- Chaléard J.-L., Planel S., Ruf T., 2017. The Oases challenged by Glocalisation. General conclusion. In Lavie E., Marshall A., Oases and globalisation, ruptures and continuities. Springer Geography, Springer, p. 243-252.

- Ruf T., 2015. Histoire des techniques hydrauliques. In Euzen A. (ed.), Jeandel C. (ed.), Mosseri R. (ed.). L'eau à découvert. Paris, CNRS, p. 164-165.

MINA KLEICHE-DRAY

Mina Kleiche, mina.kleiche@ird.fr, est historienne à l'IRD UMR CEPED. 